Discrete Comput Geom 26:105-146 (2001)

DOI: $10.1007 / \mathrm{s} 00454-001-0018-\mathrm{y}$

\title{
Reconstructing Convex Polygons in the Plane from One Directed X-Ray*
}

\author{
D. Lam $^{1}$ and D. C. Solmon ${ }^{2}$ \\ 1 c/o Student Affairs, Baylor College of Medicine, \\ Box 237, 1 Baylor Plaza, Houston, TX 77030, USA \\ d1126468@bcm.tmc.edu \\ ${ }^{2}$ Department of Mathematics, Oregon State University, \\ Corvallis, OR 97331, USA \\ solmon@math.orst.edu
}

\begin{abstract}
Gardner [7] proved that with the exception of a simple class of nonparallel wedges, convex polygons in the plane are uniquely determined by one directed X-ray. This paper develops methods for reconstructing convex polygons in the plane from one directed $\mathrm{X}$-ray. We show that nonsmooth points on the boundary of a convex body are located along rays where the derivative of the data have a jump discontinuity. Location of the nonsmooth points divides a convex polygon into a finite set of wedges. We prove uniqueness theorems and give algorithms for reconstructing nonparallel wedges from line integrals along four or more rays. Also, we characterize discrete data sets that are directed X-rays of both parallel and nonparallel wedges. Several examples of reconstructions are included.
\end{abstract}

\section{Introduction}

Since Hammer [11] posed the question of whether a convex body could be uniquely determined by a finite set of X-rays in 1961, a vast literature has developed around the question. The area of mathematics centered around answering Hammer's question has become known as geometric tomography. (The word tomography comes from the Greek tomos, meaning slice, and seems to have been borrowed from the field of X-ray computed tomography which developed parallel to work on Hammer's geometric problem over the same period of years.) See Gardner's book [9] for a comprehensive exposition of results in the area as well as interesting comments on its history.

Problems in geometric tomography have usually been divided into two classes; parallel beam geometry, where it is assumed that an X-ray consists of integrals over all

* This research was supported in part by NSF Grant DMS 9531576. 
lines parallel to a fixed direction, and fan beam or cone beam geometry, where an X-ray consists of integrals over all rays emanating from a fixed source. The main uniqueness theorems in the parallel beam case are due to Gardner and McMullen [10]. In this paper we only deal with fan beam geometry, and following the terminology of [9] refer to these as directed X-rays. Falconer [5], [6] and Gardner [7] proved that a convex body is uniquely determined by directed X-rays from two sources provided the line that passes through the sources meets the convex body. Volčič [18] showed that directed X-rays from any three noncollinear sources uniquely determine a convex body. See Chapter 5, and notes following, of [9] for extensions of these results and related questions.

While there is extensive literature on the uniqueness question little has been done on producing algorithms for reconstruction. Chang and Chow [1] and Chang and Wang [2] have used convex approximations and two orthogonal parallel X-ray sets in a medical application. Kölzow et al. [12] have also worked on reconstructions in the parallel beam case from a few directions. Edelsbrunner and Skiena [4] devised a method to reconstruct convex polygons successively from three sets of parallel X-rays. More about reconstruction in the parallel case can be found in Chapter 1, and the extensive notes following, in [9]. Almost nothing has been done on reconstruction in the case of directed X-rays. Falconer [5] points out that his proof is constructive and could lead to an algorithm for reconstruction. In [3] Dartmann implements a reconstruction algorithm based on Falconer's proof. Dartmann succeeds in locating the base points where the line passing through the sources meets the convex body, but needs information beyond the directed $\mathrm{X}$-ray data to proceed further.

In this paper we address the problem of reconstructing convex polygons in the plane from a single directed X-ray from a source outside of the polygon. That this might be possible was suggested by a result of Gardner. In [8] he proves that convex polygons in the plane are uniquely determined by two directed X-rays, and with the exception of a simple class of polygons called parallel wedges, one directed X-ray will suffice. As is much of the work in geometric tomography, the proof in [8], see also [9], is elegant, but does not lend itself immediately to a reconstruction algorithm. Throughout this paper we forego elegance in favor of results and techniques that lend themselves to computation and, in particular, reconstruction.

The paper proceeds as follows. The first section is used to establish notation and give some background results. In reconstructing a convex body on a computer, one deals with a finite number of rays. We introduce a quadratic form that is used throughout and is particularly useful in characterizing when a discrete set of directed X-ray data is directed $\mathrm{X}$-ray data of a convex body. In fact, we use the quadratic form to prove Longinetti's theorem [16] that the graph of a directed X-ray of a convex body lies on the boundary of a convex body. In the second section we show that rays along which nonsmooth points of the boundary of a convex body lie can be detected from a single directed X-ray, and derive geometric information about the nonsmooth point from the left and right derivatives of the directed X-rays through such a point. In [5] Falconer obtained extensive information about the qualitative relationship between the smoothness of the convex body and the smoothness of the directed X-rays.

From this point on we turn our attention to polygons. Location of the nonsmooth points, vertices of a polygon, divides a polygon into a finite set of quadrilaterals or triangles, called wedges. Much of the rest of the paper is devoted to finding algorithms 
for reconstructing wedges and characterizing data that come from a wedge. In Section 3 we investigate what information can be determined about a wedge from three rays, that is, from the integrals over three half-lines emanating from a fixed source. We show that three rays are sufficient to distinguish a parallel wedge from other convex bodies. The slope of the parallel sides can be determined but not the location of the wedge [8]. A characterization of sets of three data points that can be directed X-rays of a nonparallel wedge is given, and it is shown that there is a one-parameter family of nonparallel wedges having three rays in common. A general method for constructing nonparallel wedges with three rays in common is given and examples are constructed. Next we prove that a nonparallel wedge is uniquely determined by its integrals over four rays and outline an algorithm for reconstruction that would use a nonlinear least squares subroutine. In Section 5 we derive an algebraic method for reconstructing a nonparallel wedge from its integrals over four rays. In Section 6 we give necessary and sufficient conditions that a finite data set of four or more points be directed X-ray data of a nonparallel wedge. In Section 7 we use the results of the previous sections to reconstruct several polygons. While the work in this paper is directed at reconstructing convex polygons, the method can be adapted to some nonconvex polygons. One reconstruction is of a nonconvex polygon where the vertices and source align in such a way that the directed X-ray data are smooth in a neighborhood of the ray passing through them. Nevertheless, these vertices are located using the consistency conditions for directed X-ray data of a wedge.

Around 1990, Gardner (personal communication) proved both the four-rays theorem for nonparallel wedges and that rays along which vertices of a polygon lie can be detected by jumps in the derivative of the directed X-ray data, but never published the results. The first result is Theorem 4.1 below, while the second is a special case of Theorem 2.4.

\section{Notation and Fundamentals}

Throughout $K$ denotes a convex body in the plane, that is, $K$ is a compact, convex subset of the plane with nonempty interior. The characteristic function of $K$ is denoted $\chi_{K}$. The unit circle in the plane centered at the origin is denoted $S^{1}$. Points on the unit circle, denoted $\theta$, are called directions. We often write $\theta=(\cos \varphi, \sin \varphi)$, where the angle $\varphi$ is measured counterclockwise from the positive $x$-axis. Polar coordinates of a point in the plane are written $(r, \varphi)$, where we always assume that $r \geq 0$.

Definition 1.1. The directed X-ray of the convex body $K$ from source point $a$ in the direction $\theta=(\cos \varphi, \sin \varphi)$ is defined to be

$$
\mathcal{D}_{a} \chi_{K}(\theta)=\int_{0}^{\infty} \chi_{K}(a+t \theta) d t=\int_{0}^{\infty} \chi_{K}(a+t(\cos \varphi, \sin \varphi)) d t .
$$

The directed X-ray of $K$ is the function $\mathcal{D}_{a} \chi_{K}$ on the unit circle. Usually it is convenient to think of the directed X-ray as a function of the polar angle $\varphi$ instead of a function of the direction $\theta=(\cos \varphi, \sin \varphi)$. In this case, with some abuse of notation, we write $\mathcal{D}_{a} \chi_{K}(\varphi)$. In computed tomography the directed X-ray of $K$ is often referred to as the fan beam transform, or divergent beam transform, of the function $\chi_{K}$. 
Since we are only concerned with data obtained from a single directed X-ray of $K$ we may, without loss of generality, assume that the source point $a$ is the origin. With the source so fixed we write $\mathcal{D} \chi_{K}(\theta)$ or $\mathcal{D} \chi_{K}(\varphi)$. We always assume that the origin is not in the interior of $K$ and, with the exception of this section, that the origin is not in $K$. Since $K$ is compact if the origin is not in $K$, then it is a positive distance from $K$ and the cone generated by the origin and $K$ has vertex angle $<\pi$.

It is convenient to introduce the rotation matrix

$$
\gamma_{\alpha}=\left[\begin{array}{rr}
\cos \alpha & -\sin \alpha \\
\sin \alpha & \cos \alpha
\end{array}\right]
$$

and the rotation operator $\Gamma_{\alpha}$ which acts on functions on the plane and $S^{1}$ via

$$
\left(\Gamma_{\alpha} f\right)(x, y)=f\left(\gamma_{\alpha}(x, y)\right)=f(x \cos \alpha-y \sin \alpha, x \sin \alpha+y \cos \alpha) .
$$

Clearly $\Gamma_{\alpha}$ maps convex bodies to convex bodies and one has the following lemma, which is an analytic expression of the commutativity of rotations and the directed X-ray of a convex body with the source point at the origin. Since the result is geometrically obvious, we omit the proof.

Lemma 1.2. $\mathcal{D}\left(\Gamma_{\alpha} \chi_{K}\right)(\theta)=\Gamma_{\alpha}\left(\mathcal{D} \chi_{K}(\theta)\right)$, or in terms of the polar angle

$$
\mathcal{D}\left(\Gamma_{\alpha} \chi_{K}\right)(\varphi)=\mathcal{D} \chi_{K}(\varphi+\alpha) .
$$

The above lemma will be used to rotate the convex body and the rays along which data is taken to a position that simplifies computations.

Now suppose that for $j=1,2,3$ rays from the origin with directions $\theta_{j}=\left(\cos \varphi_{j}\right.$, $\sin \varphi_{j}$ ) meet the convex body $K$ in points $p_{j}=r_{j} \theta_{j}$ and $P_{j}=R_{j} \theta_{j}$ where $0 \leq r_{j} \leq R_{j}$. We always assume that the angles satisfy $\varphi_{1}<\varphi_{2}<\varphi_{3} \leq \varphi_{1}+\pi$, and if the origin is not a boundary point of $K$ that $\varphi_{3}<\varphi_{1}+\pi$. (For the moment we allow the origin to be a boundary point of $K$. In this case $r_{1}=r_{2}=r_{3}=0$.) Since $K$ is convex, the point $P_{2}$ either lies on the line segment from $P_{1}$ to $P_{3}$ or this segment separates $P_{2}$ from the origin. Hence the cross product $P_{1} P_{2} \times P_{1} P_{3}$ of the vectors $P_{1} P_{2}=R_{2} \theta_{2}-R_{1} \theta_{1}$ and $P_{1} P_{3}=R_{3} \theta_{3}-R_{1} \theta_{1}$ is either $O=(0,0)$ or points upward. That is $P_{1} P_{2} \times P_{1} P_{3}=C k$, with $C \geq 0$ and $k$ the unit upward pointing normal to the plane. Since $\theta_{i} \times \theta_{j}=$ $\sin \left(\varphi_{j}-\varphi_{i}\right) k, C=R_{1} R_{2} \sin \left(\varphi_{2}-\varphi_{1}\right)+R_{2} R_{3} \sin \left(\varphi_{3}-\varphi_{2}\right)-R_{1} R_{3} \sin \left(\varphi_{3}-\varphi_{1}\right)$. Similar considerations with the points $p_{j}$ give that $p_{1} p_{2} \times p_{1} p_{3}=D k$, where $D=$ $r_{1} r_{2} \sin \left(\varphi_{2}-\varphi_{1}\right)+r_{2} r_{3} \sin \left(\varphi_{3}-\varphi_{2}\right)-r_{1} r_{3} \sin \left(\varphi_{3}-\varphi_{1}\right) \leq 0$. These computations lead us to define the following quadratic form.

Definition 1.3. Given vectors $r=\left[r_{1}, r_{2}, r_{3}\right], \varphi=\left[\varphi_{1}, \varphi_{2}, \varphi_{3}\right] \in R^{3}$ we define

$$
Q(r)=Q(r, \varphi)=r A r^{T}=r_{1} r_{2} \sin \left(\varphi_{2}-\varphi_{1}\right)+r_{2} r_{3} \sin \left(\varphi_{3}-\varphi_{2}\right)-r_{1} r_{3} \sin \left(\varphi_{3}-\varphi_{1}\right),
$$

where

$$
A=\frac{1}{2}\left[\begin{array}{ccc}
0 & \sin \left(\varphi_{2}-\varphi_{1}\right) & -\sin \left(\varphi_{3}-\varphi_{1}\right) \\
\sin \left(\varphi_{2}-\varphi_{1}\right) & 0 & \sin \left(\varphi_{3}-\varphi_{2}\right) \\
-\sin \left(\varphi_{3}-\varphi_{1}\right) & \sin \left(\varphi_{3}-\varphi_{2}\right) & 0
\end{array}\right]
$$


The lemma below follows easily from the discussion above and properties of the cross product.

Lemma 1.5. Suppose that $\varphi_{1}<\varphi_{2}<\varphi_{3} \leq \varphi_{1}+\pi$ and $r_{j} \geq 0, j=1,2,3$.

(a) $Q(r)=0$ if and only if the points $\left(r_{j}, \varphi_{j}\right)$ are collinear.

(b) If $Q(r)>0$, then the line passing through $\left(r_{1}, \varphi_{1}\right)$ and $\left(r_{3}, \varphi_{3}\right)$ separates $\left(r_{2}, \varphi_{2}\right)$ from the origin. If $Q(r)<0$, then the origin and $\left(r_{2}, \varphi_{2}\right)$ lie on the same side of the line passing through $\left(r_{1}, \varphi_{1}\right)$ and $\left(r_{3}, \varphi_{3}\right)$.

(c) If for $j=1,2,3$ the rays making angle $\varphi_{j}$ with the positive $x$-axis meet the boundary of the convex body $K$ in points $\left(r_{j}, \varphi_{j}\right)$ and $\left(R_{j}, \varphi_{j}\right)$ where $0 \leq r_{j} \leq R_{j}$, then $Q(R) \geq 0$ and $Q(r) \leq 0$.

Remark 1.6. The quadratic form $Q$ will appear often. We will sometimes want to apply $Q$ to three points selected from a larger set. In this case we write $Q\left(r_{i}, r_{j}, r_{k}\right)$, where

$$
Q\left(r_{i}, r_{j}, r_{k}\right)=r_{i} r_{j} \sin \left(\varphi_{j}-\varphi_{i}\right)+r_{j} r_{k} \sin \left(\varphi_{k}-\varphi_{j}\right)-r_{i} r_{k} \sin \left(\varphi_{k}-\varphi_{i}\right),
$$

and it is understood that $Q$ is applied to the points with polar coordinates $\left(r_{i}, \varphi_{i}\right),\left(r_{j}, \varphi_{j}\right)$, and $\left(r_{k}, \varphi_{k}\right)$.

The next lemma is a special case of Theorem 5.1 .15 on p. 172 of [9] that is due to Longinetti [16]. The proof given here will be useful in establishing later results.

Lemma 1.8. Let $K$ be a convex body. Suppose that $\varphi_{1}<\varphi_{2}<\varphi_{3} \leq \varphi_{1}+\pi$ and $\mathcal{D} \chi_{K}\left(\varphi_{j}\right)=X_{j}, j=1,2,3$. Set $X=\left[X_{1}, X_{2}, X_{3}\right]$. Then $Q(X) \geq 0$. Consequently, the set $D_{K}=\left\{r \theta: 0 \leq r \leq \mathcal{D} \chi_{K}(\theta)\right\}$ is a convex body.

Proof. If the origin is a boundary point of $K$, then $D_{K}=K$ and there is nothing to prove. Assume then that the origin is not in $K$. It suffices to show that $Q(X) \geq 0$, because the convexity of $D_{K}$ will follow from Lemma 1.5(a), (b). Since $K$ is convex, we may write $X=R-r$, where $\left(r_{j}, \varphi_{j}\right)$ and $\left(R_{j}, \varphi_{j}\right), 0<r_{j} \leq R_{j}$, are the points of intersection of the three rays with $K$. By Lemma 1.5(c), $Q(R) \geq 0$ and $Q(r) \leq 0$. Consequently,

$$
\begin{aligned}
& R_{2} \geq \frac{R_{1} R_{3} \sin \left(\varphi_{3}-\varphi_{1}\right)}{R_{1} \sin \left(\varphi_{2}-\varphi_{1}\right)+R_{3} \sin \left(\varphi_{3}-\varphi_{2}\right)} \quad \text { and } \\
& r_{2} \leq \frac{r_{1} r_{3} \sin \left(\varphi_{3}-\varphi_{1}\right)}{r_{1} \sin \left(\varphi_{2}-\varphi_{1}\right)+r_{3} \sin \left(\varphi_{3}-\varphi_{2}\right)} .
\end{aligned}
$$

Now

$$
\begin{aligned}
Q(X)= & Q(R-r) \\
= & \left(R_{1}-r_{1}\right)\left(R_{2}-r_{2}\right) \sin \left(\varphi_{2}-\varphi_{1}\right)+\left(R_{2}-r_{2}\right)\left(R_{3}-r_{3}\right) \sin \left(\varphi_{3}-\varphi_{2}\right) \\
& -\left(R_{1}-r_{1}\right)\left(R_{3}-r_{3}\right) \sin \left(\varphi_{3}-\varphi_{1}\right) .
\end{aligned}
$$


From the inequalities (1.9) we have that

$$
\begin{aligned}
& R_{2}-r_{2} \geq \frac{R_{1} R_{3} \sin \left(\varphi_{3}-\varphi_{1}\right)}{R_{1} \sin \left(\varphi_{2}-\varphi_{1}\right)+R_{3} \sin \left(\varphi_{3}-\varphi_{2}\right)}-\frac{r_{1} r_{3} \sin \left(\varphi_{3}-\varphi_{1}\right)}{r_{1} \sin \left(\varphi_{2}-\varphi_{1}\right)+r_{3} \sin \left(\varphi_{3}-\varphi_{2}\right)} \\
&=\sin \left(\varphi_{3}-\varphi_{1}\right) \\
& \times \frac{R_{1} R_{3}\left[r_{1} \sin \left(\varphi_{2}-\varphi_{1}\right)+r_{3} \sin \left(\varphi_{3}-\varphi_{2}\right)\right]-r_{1} r_{3}\left[R_{1} \sin \left(\varphi_{2}-\varphi_{1}\right)+R_{3} \sin \left(\varphi_{3}-\varphi_{2}\right)\right]}{\left[R_{1} \sin \left(\varphi_{2}-\varphi_{1}\right)+R_{3} \sin \left(\varphi_{3}-\varphi_{2}\right)\right]\left[r_{1} \sin \left(\varphi_{2}-\varphi_{1}\right)+r_{3} \sin \left(\varphi_{3}-\varphi_{2}\right)\right]} .
\end{aligned}
$$

Replace $R_{2}-r_{2}$ in (1.10) by the last expression. Some elementary, but tedious, algebra (which we omit) leads to

$$
\begin{aligned}
Q(X) & =Q(R-r) \\
& \geq \frac{\left(r_{1} R_{3}-r_{3} R_{1}\right)^{2} \sin \left(\varphi_{2}-\varphi_{1}\right) \sin \left(\varphi_{3}-\varphi_{2}\right) \sin \left(\varphi_{3}-\varphi_{1}\right)}{\left[R_{1} \sin \left(\varphi_{2}-\varphi_{1}\right)+R_{3} \sin \left(\varphi_{3}-\varphi_{2}\right)\right]\left[r_{1} \sin \left(\varphi_{2}-\varphi_{1}\right)+r_{3} \sin \left(\varphi_{3}-\varphi_{2}\right)\right]} \\
& \geq 0 .
\end{aligned}
$$

The following corollary to the proof is used later.

Lemma 1.11. Assume that $r, R$, and $X$ are as in (1.10). Then $Q(X)=Q(R-r)=0$ if and only if $Q(R)=Q(r)=0$ and $r_{1} R_{3}-r_{3} R_{1}=0$.

Proof. From the proof of Lemma 1.8, $Q(X)=Q(R-r)=0$ if and only if $r_{1} R_{3}-$ $r_{3} R_{1}=0$ and equality holds in both inequalities in (1.9). The latter hold if and only if $Q(R)=Q(r)=0$.

We need one more result concerning the quadratic form $Q$.

Lemma 1.12. Let $\varphi_{1}<\varphi_{2}<\cdots<\varphi_{n}<\varphi_{1}+\pi$ and $X_{j}>0, j=1,2, \ldots, n$. Suppose that for each index $j, 1 \leq j \leq n-2, Q\left(X_{j}, X_{j+1}, X_{j+2}\right) \geq 0$. Then $Q\left(X_{i}, X_{j}, X_{k}\right)$ $\geq 0$ whenever $1 \leq i<j<k \leq n$. In particular, the set of points with polar coordinates $\left(X_{j}, \varphi_{j}\right), j=1, \ldots, n$, lie on a convex body having the origin as a boundary point if and only if $Q\left(X_{j}, X_{j+1}, X_{j+2}\right) \geq 0$, for $j=1,2, \ldots, n-2$.

This result can be proven by induction. We omit the proof in favor of a geometric formulation which makes the lemma obvious. Indeed, for $j=1,2, \ldots, n$ let $P_{j}=$ $\left(X_{j} \cos \varphi_{j}, X_{j} \sin \varphi_{j}\right)$ and $O=(0,0)$. The lemma then states: "If for each consecutive triple of points $P_{j}, P_{j+1}, P_{j+2}$, the polygon $O, P_{j}, P_{j+1}, P_{j+2}, O$ (obtained by connecting consecutive points with a straight line) is convex, then the polygon $O, P_{1}, \ldots, P_{n}, O$ is convex." (Recall that $\varphi_{1}<\varphi_{2}<\cdots<\varphi_{n}<\varphi_{1}+\pi$.)

Finally we record two elementary trigonometric identities which will be used frequently:

$$
\begin{aligned}
& \sin (\alpha-\beta) \sin (\gamma-\delta)-\sin (\alpha-\delta) \sin (\gamma-\beta)=\sin (\alpha-\gamma) \sin (\beta-\delta) \\
& \sin (\alpha-\beta) \sin (\gamma-\delta)-\sin (\alpha-\gamma) \sin (\beta-\delta)=\sin (\alpha-\delta) \sin (\gamma-\beta)
\end{aligned}
$$

These follow easily from the identity $\sin (A) \sin (B)=(\cos (A-B)-\cos (A+B)) / 2$. 


\section{Detecting Nonsmooth Points}

Throughout the rest of the paper we assume that the origin is not in $K$. With an appropriate rotation of coordinates, in any sufficiently small neighborhood of a boundary point of $K$, the boundary of $K, \partial K$, can be described by a convex (or concave) function. Hence with the possible exception of an at most countable set of points, if $Q \in \partial K$ there exists a unique tangent line to $\partial K$ at $Q$. Points in $\partial K$ at which there does not exist a unique tangent line will be referred to as nonsmooth points. We will show that with the possible exception of supporting rays, a directed X-ray of $K$ determines the rays on which nonsmooth points lie and investigate the geometric information contained in the left and right derivatives of the directed X-ray data at the nonsmooth points.

The following well-known result on convex and concave functions provides the basis for detecting nonsmooth points.

Theorem 2.1 [17, p. 113]. If $f$ is a convex (concave) function on the interval $(a, b)$, then $f$ is absolutely continuous on each closed subinterval of $(a, b)$. The right- and lefthand derivatives of $f$ exist at each point of $(a, b)$ and are equal to each other except on a countable set. The left- and right-hand derivatives are increasing (decreasing) functions, and at each point the left-hand derivative is less than or equal to (greater than or equal to) the right-hand derivative.

Suppose that $F$ is a positive convex or concave function defined on $(-a, a)$. If $(x, y)$ is a point on the graph of $F$, we may write $x=R(\varphi) \cos \varphi, y=R(\varphi) \sin \varphi$ where $R(\varphi)$ is the distance from $(x, y)$ to the origin. By the chain rule,

$$
F^{\prime}(x)=\frac{d y}{d x}=\frac{d y / d \varphi}{d x / d \varphi}=\frac{R^{\prime}(\varphi) \sin \varphi+R(\varphi) \cos \varphi}{R^{\prime}(\varphi) \cos \varphi-R(\varphi) \sin \varphi}
$$

at the smooth points. The identity also holds at nonsmooth points provided the derivatives are replaced by either the right- or left-hand derivatives throughout. In particular we have for the "right"- and "left"-hand derivatives $F_{+}^{\prime}, F_{-}^{\prime}$, respectively

$$
\begin{aligned}
& F_{+}^{\prime}(x)=\frac{R_{-}^{\prime}(\varphi) \sin \varphi+R(\varphi) \cos \varphi}{R_{-}^{\prime}(\varphi) \cos \varphi-R(\varphi) \sin \varphi} \quad \text { and } \\
& F_{-}^{\prime}(x)=\frac{R_{+}^{\prime}(\varphi) \sin \varphi+R(\varphi) \cos \varphi}{R_{+}^{\prime}(\varphi) \cos \varphi-R(\varphi) \sin \varphi} .
\end{aligned}
$$

Due to the standard counterclockwise orientation of angles in the plane there is some ambiguity in the terms "left" and "right" derivative. For any function of a real variable, we adopt the notation

$$
f_{+}^{\prime}(x)=\lim _{\substack{h \rightarrow 0 \\ h>0}} \frac{f(x+h)-f(x)}{h} \quad \text { and } \quad f_{-}^{\prime}(x)=\lim _{\substack{h \rightarrow 0 \\ h<0}} \frac{f(x+h)-f(x)}{h} .
$$

This explains the derivatives $R_{-}^{\prime}$ and $R_{+}^{\prime}$ on the right-hand side of (2.2) above. Solving for $R_{-}^{\prime}$ and $R_{+}^{\prime}$ in (2.2) gives

$$
R_{-}^{\prime}(\varphi)=R(\varphi) \frac{F_{+}^{\prime}(x) \sin \varphi+\cos \varphi}{F_{+}^{\prime}(x) \cos \varphi-\sin \varphi} \text { and } R_{+}^{\prime}(\varphi)=R(\varphi) \frac{F_{-}^{\prime}(x) \sin \varphi+\cos \varphi}{F_{-}^{\prime}(x) \cos \varphi-\sin \varphi}
$$


Theorem 2.4. Let $P$ be a point on $\partial K$. Suppose that the ray $\varphi=\varphi_{0}$ passes through the interior of $K$ and contains $P$. Then $P$ is a nonsmooth point of $\partial K$ if and only if $\left(\mathcal{D} \chi_{K}\right)_{-}^{\prime}\left(\varphi_{0}\right)-\left(\mathcal{D} \chi_{K}\right)_{+}^{\prime}\left(\varphi_{0}\right)>0$.

Proof. Consider a ray $\varphi=\varphi_{0}$ that passes through the interior of $K$ and intersects $\partial K$ in points $q, Q$, with $q$ being the closer to the origin. Since rotations commute with directed $\mathrm{X}$-rays, Lemma 1.2, we may rotate coordinates so that in a neighborhood of $q, \partial K$ can be described in rectangular and polar coordinates as

$$
y=f(x), \quad x=r(\varphi) \cos \varphi, \quad y=r(\varphi) \sin \varphi, \quad \text { with } f \text { a convex function, }
$$

and in a neighborhood of $Q, \partial K$ can be described as

$$
y=F(x), \quad x=R(\varphi) \cos \varphi, \quad y=R(\varphi) \sin \varphi, \quad \text { with } F \text { a concave function. }
$$

We then have $\mathcal{D} \chi_{K}(\varphi)=R(\varphi)-r(\varphi)$ for rays near $\varphi_{0}$. Supposing now that the $x$ coordinates of $q$ and $Q$ are $s_{0}$ and $t_{0}$, respectively, we obtain, from (2.2),

$$
\begin{aligned}
& \left(\mathcal{D} \chi_{K}\right)_{-}^{\prime}\left(\varphi_{0}\right)=R\left(\varphi_{0}\right) \frac{F_{+}^{\prime}\left(t_{0}\right) \sin \varphi_{0}+\cos \varphi_{0}}{F_{+}^{\prime}\left(t_{0}\right) \cos \varphi_{0}-\sin \varphi_{0}}-r\left(\varphi_{0}\right) \frac{f_{+}^{\prime}\left(s_{0}\right) \sin \varphi_{0}+\cos \varphi_{0}}{f_{+}^{\prime}\left(s_{0}\right) \cos \varphi_{0}-\sin \varphi_{0}}, \\
& \left(\mathcal{D} \chi_{K}\right)_{+}^{\prime}\left(\varphi_{0}\right)=R\left(\varphi_{0}\right) \frac{F_{-}^{\prime}\left(t_{0}\right) \sin \varphi_{0}+\cos \varphi_{0}}{F_{-}^{\prime}\left(t_{0}\right) \cos \varphi_{0}-\sin \varphi_{0}}-r\left(\varphi_{0}\right) \frac{f_{-}^{\prime}\left(s_{0}\right) \sin \varphi_{0}+\cos \varphi_{0}}{f_{-}^{\prime}\left(s_{0}\right) \cos \varphi_{0}-\sin \varphi_{0}} .
\end{aligned}
$$

Consequently,

$$
\begin{aligned}
\left(\mathcal{D} \chi_{K}\right)_{-}^{\prime}\left(\varphi_{0}\right)- & \left(\mathcal{D} \chi_{K}\right)_{+}^{\prime}\left(\varphi_{0}\right) \\
= & \frac{R\left(\varphi_{0}\right)\left(F_{-}^{\prime}\left(t_{0}\right)-F_{+}^{\prime}\left(t_{0}\right)\right)}{\left[F_{+}^{\prime}\left(t_{0}\right) \cos \varphi_{0}-\sin \varphi_{0}\right]\left[F_{-}^{\prime}\left(t_{0}\right) \cos \varphi_{0}-\sin \varphi_{0}\right]} \\
& +\frac{r\left(\varphi_{0}\right)\left(f_{+}^{\prime}\left(s_{0}\right)-f_{-}^{\prime}\left(s_{0}\right)\right)}{\left[f_{+}^{\prime}\left(s_{0}\right) \cos \varphi_{0}-\sin \varphi_{0}\right]\left[f_{-}^{\prime}\left(t_{0}\right) \cos \varphi_{0}-\sin \varphi_{0}\right]} .
\end{aligned}
$$

If we now choose coordinates so that $\varphi_{0}=\pi / 2$ and hence $s_{0}=t_{0}=0$, then we obtain

$\left(\mathcal{D} \chi_{K}\right)_{-}^{\prime}(\pi / 2)-\left(\mathcal{D} \chi_{K}\right)_{+}^{\prime}(\pi / 2)=R(\pi / 2)\left[F_{-}^{\prime}(0)-F_{+}^{\prime}(0)\right]+r(\pi / 2)\left[f_{+}^{\prime}(0)-f_{-}^{\prime}(0)\right]$.

Since $F$ is convex and $f$ is concave, it follows from Theorem 2.1 that $F_{-}^{\prime}(0)-F_{+}^{\prime}(0) \geq 0$ and $f_{+}^{\prime}(0)-f_{-}^{\prime}(0) \geq 0$. Since $R(\pi / 2)>r(\pi / 2)>0, \mathcal{D} \chi_{K}$ is differentiable at $\pi / 2$ if and only if both $F$ and $f$ are differentiable at 0 , that is, if and only if $Q$ and $q$ are smooth points of $\partial K$. Conversely, $\mathcal{D} \chi_{K}$ is not differentiable at $\varphi_{0}$ if and only if one or both of these points are nonsmooth points of $\partial K$.

Falconer [5] has shown that nonsmoothness along supporting rays cannot be detected from one directed X-ray.

Remark. In reconstructing convex polygons from a finite set of rays from the origin, one can use first-order divided differences to approximate $\left(\mathcal{D} \chi_{K}\right)_{-}^{\prime}$ and $\left(\mathcal{D} \chi_{K}\right)_{+}^{\prime}$. As is 
predicted by (2.5) significant jumps in the divided differences occur as the discrete data crosses a ray on which one or two nonsmooth points lie.

The geometric information contained in (2.5) can also be expressed nicely in terms of the angles of inclination of the left and right tangent lines at the nonsmooth points. If we write

$\tan \omega_{+}=F_{+}^{\prime}\left(t_{0}\right), \quad \tan \omega_{-}=F_{-}^{\prime}\left(t_{0}\right), \quad \tan \Psi_{+}=f_{+}^{\prime}\left(s_{0}\right), \quad \tan , \Psi_{-}=f_{-}^{\prime}\left(s_{0}\right)$, then (2.5) becomes

$$
\begin{aligned}
\left(\mathcal{D} \chi_{K}\right)_{-}^{\prime}\left(\varphi_{0}\right)-\left(\mathcal{D} \chi_{K}\right)_{+}^{\prime}\left(\varphi_{0}\right)= & R\left(\varphi_{0}\right) \frac{\sin \left(\omega_{-}-\omega_{+}\right)}{\sin \left(\varphi_{0}-\omega_{+}\right) \sin \left(\varphi_{0}-\omega_{-}\right)} \\
& +r\left(\varphi_{0}\right) \frac{\sin \left(\Psi_{-}-\Psi_{+}\right)}{\sin \left(\varphi_{0}-\Psi_{+}\right) \sin \left(\varphi_{0}-\Psi_{-}\right)} .
\end{aligned}
$$

The assumption that $K$ is convex is not needed in the derivation of (2.5). In fact (2.5) shows that in the nonconvex case, $\mathcal{D} \chi_{K}$ may be smooth at a point $\varphi_{0}$ even though two nonsmooth points of $\partial K$ lie on the ray $\varphi=\varphi_{0}$. In most cases there is only one vertex along a ray and one of the two terms in the sum is zero. Unfortunately, without some additional a priori information we cannot determine which one.

Remark. Volčič [18] has shown that a convex body in the plane is uniquely determined from its directed X-rays from any three noncollinear sources outside of the convex body. However, the proof does not lead to a method of reconstruction. The above results lead to a method that will successively locate nonsmooth points on the boundary of a convex body from three sources outside of $K$. (The concept of successive determination was introduced by Edelsbrunner and Skiena [4] in reconstructing convex polygons with parallel X-rays.) Indeed, suppose that $a$ and $b$ are two sources outside of $K$. Rays from $a$ and $b$ on which nonsmooth boundary points of $K$ lie can be detected by looking for discontinuities in the derivative of the directed X-rays. The nonsmooth points of $K$ are a subset of the intersection points of the rays containing nonsmooth points from the two sources. Now pick a third source point $c$ that lies outside of $K$ and does not lie on a line containing two intersection points. (As the set of nonsmooth points is countable, such a choice is always possible. The set of lines containing two possible nonsmooth points is also countable and hence has measure zero.) Find rays from $c$ on which nonsmooth points of the boundary of $K$ lie. The points of intersection of rays from $a, b, c$, all of which contain nonsmooth points of the boundary of $K$, are nonsmooth points. Perhaps this could be used to approximate the location of nonsmooth points on the boundary of a convex body. Then a reconstruction might be possible from knowledge of the nonsmooth points and the directed X-ray data from the three points. Of course, the convex body need not have any nonsmooth points.

\section{Wedges and Three Rays}

By a wedge $W$ we mean a quadrilateral (or triangle) with all the vertices of $W$ lying on two rays emanating from a common point, the $\mathrm{X}$-ray source. Without loss of generality 
we choose coordinates so that the source is at the origin and the rays are given by $\varphi=\alpha, \varphi=\beta$, with $0<\beta-\alpha<\pi$. Further, we always assume that the wedge $W$ is a positive distance from the origin. The sides of $W$ then consist of segments of the rays $\varphi=\alpha, \varphi=\beta$ (possibly degenerate in the case of a triangle) and segments of two lines $L_{m}, L_{M}$ having slopes $m$ and $M$, respectively. The lines $L_{m}, L_{M}$ may intersect on the rays but do not intersect in the interior of the cone $C(\alpha, \beta)=\{(r, \varphi): r \geq 0$ and $\alpha \leq \varphi \leq \beta\}$. If the lines $L_{m}, L_{M}$ are parallel, $W$ is called a parallel wedge. In this section we assess the information determined about convex bodies $K$, and wedges $W$, from knowledge of the integrals along three rays making angles $\alpha \leq \varphi_{1}<\varphi_{2}<\varphi_{3} \leq \beta$. The symbols $X, r, R$ stand for vectors in $R^{3}$. To say that $X>O$ means that each of the components of the vector is positive. Frequent use is made of the quadratic form $Q$ and matrix $A$ defined in (1.3), (1.4).

It is well known that a parallel wedge cannot be reconstructed from one directed X-ray. Indeed, suppose that a parallel wedge $W$ has vertices $\left(r_{\alpha}, \alpha\right),\left(R_{\alpha}, \alpha\right),\left(r_{\beta}, \beta\right),\left(R_{\beta}, \beta\right)$ with $0<r_{\alpha}<R_{\alpha}, 0<r_{\beta}<R_{\beta}$. If $a \geq-r_{\alpha}, b \geq-r_{\beta}$, and $\left(r_{\alpha}+a\right) r_{\beta}=\left(r_{\beta}+b\right) r_{\alpha}$, then the wedge $W_{a, b}$ with vertices $\left(r_{\alpha}+a, \alpha\right),\left(R_{\alpha}+a, \alpha\right),\left(r_{\beta}+b, \beta\right),\left(R_{\beta}+b, \beta\right)$ is a parallel wedge with the same directed X-rays as $W$ for any angle $\varphi$ such that $\alpha \leq \varphi \leq \beta$. See [8] and [9].

The next result shows that three rays suffice to determine whether a convex body contains a parallel wedge.

Theorem 3.1. Suppose that $K$ is a convex body such that $\mathcal{D} \chi_{K}\left(\varphi_{j}\right)=X_{j} \geq 0$ for $j=1,2,3$ where $\alpha \leq \varphi_{1}<\varphi_{2}<\varphi_{3} \leq \beta$. Let $X=\left(X_{1}, X_{2}\right.$, $\left.X_{3}\right)$. If $Q(X)=0$, then the intersection of $K$ with the cone $C\left(\varphi_{1}, \varphi_{3}\right)$ is a parallel wedge and the lines $L_{m}, L_{M}$ bounding the parallel wedge have common slope $\left(X_{3} \sin \varphi_{3}-X_{1} \sin \varphi_{1}\right) /\left(X_{3} \cos \varphi_{3}-\right.$ $X_{1} \cos \varphi_{1}$ ) (the lines being vertical when $X_{3} \cos \varphi_{3}-X_{1} \cos \varphi_{1}=0$ ). Nothing can be determined about the distance of the parallel wedge from the origin even if additional rays from the region $\varphi_{1} \leq \varphi \leq \varphi_{3}$ are included. In particular, if $K$ is a wedge, it is a parallel wedge.

Proof. For $j=1,2,3$, let $r=\left(r_{1}, r_{2}, r_{3}\right)$ and $R=\left(R_{1}, R_{2}, R_{3}\right)$, where $0<r_{j} \leq R_{j}$, be the points of intersection of the ray $\varphi=\varphi_{j}$ with $K$. Then $X=R-r$. Suppose that $Q(X)=0$. By Lemma $1.11 Q(r)=Q(R)=0$ and $r_{1} R_{3}-r_{3} R_{1}=0$. By Lemma $1.5(\mathrm{a})$ the points $r_{j} \theta_{j}$ are collinear and the points $R_{j} \theta_{j}$ are collinear. Hence the intersection of $K$ with the cone $C\left(\varphi_{1}, \varphi_{3}\right)$ is a wedge. It remains to show that the intersection is a parallel wedge. Since $r_{1} R_{3}-r_{3} R_{1}=0, r_{1} / R_{1}=r_{3} / R_{3}$. Hence the triangles with vertices $O, r_{1} \theta_{1}, r_{3} \theta_{3}$ and $O, R_{1} \theta_{1}, R_{3} \theta_{3}$ are similar. So, the rays from $r_{1} \theta_{1}$ to $r_{3} \theta_{3}$ and from $R_{1} \theta_{1}$ to $R_{3} \theta_{3}$ are parallel and $K$ is a parallel wedge. Since $X_{j}=R_{j}-r_{j}, X_{1} / X_{3}=$ $r_{1} / r_{3}=R_{1} / R_{3}$. Hence the common slope of lines $L_{m}$ and $L_{M}$ is

$$
\frac{r_{3} \sin \varphi_{3}-r_{1} \sin \varphi_{1}}{r_{3} \cos \varphi_{3}-r_{1} \cos \varphi_{1}}=\frac{X_{3} \sin \varphi_{3}-X_{1} \sin \varphi_{1}}{X_{3} \cos \varphi_{3}-X_{1} \cos \varphi_{1}} .
$$

We now turn our attention to the question of what can be deduced about nonparallel wedges from three rays. If $K=W$ is a nonparallel wedge and $r, R$, and $X=R-r$ are as in the theorem, then from collinearity $Q(r)=Q(R)=0$, while $Q(X)>0$ by 
Lemma 1.11. Hence

$$
0=Q(R)=(r+X) A(r+X)^{T}=Q(r)+2 r A X^{T}+Q(X),
$$

and consequently $r A X^{T}=-Q(X) / 2$. As the steps in the last computation are reversible, we have established the following result.

Theorem 3.2. Given $\left(X_{j}, \varphi_{j}\right), j=1,2,3$, with each $X_{j} \geq 0$ and $\varphi_{1}<\varphi_{2}<\varphi_{3}<$ $\varphi_{1}+\pi$, necessary and sufficient conditions that there exists a nonparallel wedge $W$ (bounded away from the origin) such that $\mathcal{D} \chi_{W}\left(\varphi_{j}\right)=X_{j} \geq 0$ for $j=1,2,3$ are that $Q(X)>0$ and there exists an $r>O$ which satisfies the system of equations

$$
Q(r)=0 \quad \text { and } \quad r A X^{T}=\frac{-Q(X)}{2} .
$$

Next we make the conditions in (3.3) more explicit. For simplicity, we assume that the angles the X-ray directions make with the positive $\mathrm{x}$-axis are positive but less than $\pi$.

Theorem 3.4. Let data points $\left(X_{j}, \varphi_{j}\right), j=1,2,3$, be given with each $X_{j} \geq 0$ and $0<$ $\varphi_{1}<\varphi_{2}<\varphi_{3}<\pi$. Necessary and sufficient conditions that there exist a nonparallel wedge $W$ such that $\mathcal{D} \chi_{W}\left(\varphi_{j}\right)=X_{j}$ for $j=1,2,3$ are that

$$
Q(X)>0,
$$

and there exists $a \Psi$ which satisfies

$$
0<\Psi<\varphi_{1} \quad \text { or } \quad \varphi_{3}<\Psi<\pi
$$

and

$$
\begin{aligned}
T(\Psi):= & X_{1} \sin ^{2}\left(\Psi-\varphi_{1}\right) \sin \left(\varphi_{3}-\varphi_{2}\right)-X_{2} \sin ^{2}\left(\Psi-\varphi_{2}\right) \sin \left(\varphi_{3}-\varphi_{1}\right) \\
& +X_{3} \sin ^{2}\left(\Psi-\varphi_{3}\right) \sin \left(\varphi_{2}-\varphi_{1}\right)>0
\end{aligned}
$$

Moreover, when these conditions are satisfied, then for each $\Psi$ satisfying (3.6) and (3.7) there is a unique wedge $W=W_{\Psi}$ whose near boundary line has angle of inclination $\Psi$ such that $\mathcal{D} \chi_{W}\left(\varphi_{j}\right)=X_{j}$ for $j=1,2,3$. The points of intersection $\left(r_{j}, \varphi_{j}\right)$ of the near boundary line with $W_{\Psi}$ are given by

$$
\begin{aligned}
& r_{1}=\frac{Q(X) \sin \left(\Psi-\varphi_{2}\right) \sin \left(\Psi-\varphi_{3}\right)}{T(\Psi)}, \\
& r_{j}=\frac{\sin \left(\Psi-\varphi_{1}\right)}{\sin \left(\Psi-\varphi_{j}\right)} r_{1} \quad \text { for } \quad j=2,3 .
\end{aligned}
$$

Proof. Assume that the data $\left(X_{j}, \varphi_{j}\right), j=1,2,3$, are directed X-rays of a nonparallel wedge $W$. Suppose that the near line $L_{m}$ has slope $m=\tan \Psi$ and assume for the moment 
that $\Psi \neq \pi / 2$ so that $m$ is finite. Since the points $\left(r_{j}, \varphi_{j}\right), j=1,2,3$, all lie on a line with slope $m$ it follows that

$$
m=\frac{r_{2} \sin \varphi_{2}-r_{1} \sin \varphi_{1}}{r_{2} \cos \varphi_{2}-r_{1} \cos \varphi_{1}}=\frac{r_{3} \sin \varphi_{3}-r_{1} \sin \varphi_{1}}{r_{3} \cos \varphi_{3}-r_{1} \cos \varphi_{1}} .
$$

Solving for $r_{2}$ in terms of $r_{1}$ and using that $m=\tan \Psi=(\sin \Psi) /(\cos \Psi)$ it follows that

$$
r_{j}=\frac{m \cos \varphi_{1}-\sin \varphi_{1}}{m \cos \varphi_{j}-\sin \varphi_{j}} r_{1}=\frac{\sin \left(\Psi-\varphi_{1}\right)}{\sin \left(\Psi-\varphi_{j}\right)} r_{1}, \quad \text { for } \quad j=2,3 .
$$

This establishes (3.9) when the line $L_{m}$ is not vertical. However, (3.9) now holds when $L_{m}$ is vertical by continuity. Also, since $r>O$, we must have that $\sin \left(\Psi-\varphi_{1}\right) / \sin \left(\Psi-\varphi_{j}\right)>$ 0 . Since $0<\varphi_{1}<\varphi_{2}<\varphi_{3}<\pi$ this is equivalent to (3.6). The necessity of (3.7) follows from (3.8) since the numerator in (3.8) is positive by (3.5) and (3.6). To obtain (3.8) note that from Theorem 3.2 $r A X^{T}=-Q(X) / 2$. Use (3.9) to write $r_{2}$ and $r_{3}$ in terms of $r_{1}$, substitute into this identity and solve for $r_{1}$. The result is (3.8).

To complete the proof suppose that (3.5)-(3.7) hold and fix a $\Psi$ satisfying (3.6) and (3.7). Next define $r_{1}$ by (3.8) and then $r_{2}, r_{3}$ by (3.9). Conditions (3.5)-(3.7) ensure that the $r_{j}$ are positive. The proof is completed by showing that (3.3) is satisfied. One readily checks that $Q(r)=0$. To see that $r A X^{T}=-Q(X) / 2$, let $T(\Psi)$ be the expression in (3.7). Then (3.8) and (3.9) give

$$
\begin{gathered}
r_{1}=\frac{Q(X) \sin \left(\Psi-\varphi_{2}\right) \sin \left(\Psi-\varphi_{3}\right)}{T(\Psi)}, \quad r_{2}=\frac{Q(X) \sin \left(\Psi-\varphi_{1}\right) \sin \left(\Psi-\varphi_{3}\right)}{T(\Psi)}, \\
r_{3}=\frac{Q(X) \sin \left(\Psi-\varphi_{1}\right) \sin \left(\Psi-\varphi_{2}\right)}{T(\Psi)} .
\end{gathered}
$$

Then using (1.4) and the identities (1.13) and (1.14) one obtains

$$
\begin{aligned}
& r A X^{T}=\frac{1}{2}[ X_{1}\left(r_{2} \sin \left(\varphi_{2}-\varphi_{1}\right)-r_{3} \sin \left(\varphi_{3}-\varphi_{1}\right)\right) \\
&+X_{2}\left(r_{1} \sin \left(\varphi_{2}-\varphi_{1}\right)+r_{3} \sin \left(\varphi_{3}-\varphi_{2}\right)\right) \\
&\left.+X_{3}\left(r_{2} \sin \left(\varphi_{3}-\varphi_{2}\right)-r_{1} \sin \left(\varphi_{3}-\varphi_{1}\right)\right)\right] \\
&=\frac{Q(X)}{2 T(\Psi)}[ X_{1} \sin \left(\Psi-\varphi_{1}\right)\left(\sin \left(\Psi-\varphi_{3}\right) \sin \left(\varphi_{2}-\varphi_{1}\right)\right. \\
&\left.-\sin \left(\Psi-\varphi_{2}\right) \sin \left(\varphi_{3}-\varphi_{1}\right)\right)+X_{2} \sin \left(\Psi-\varphi_{2}\right) \\
& \quad \times\left(\sin \left(\Psi-\varphi_{3}\right) \sin \left(\varphi_{2}-\varphi_{1}\right)\right. \\
&\left.\quad-\sin \left(\Psi-\varphi_{1}\right) \sin \left(\varphi_{2}-\varphi_{3}\right)\right)+X_{3} \sin \left(\Psi-\varphi_{3}\right) \\
&\left.\quad \times\left(\sin \left(\Psi-\varphi_{1}\right) \sin \left(\varphi_{3}-\varphi_{2}\right)-\sin \left(\Psi-\varphi_{2}\right) \sin \left(\varphi_{3}-\varphi_{1}\right)\right)\right] \\
&=\frac{Q(X)}{2 T(\Psi)}\left[-X_{1} \sin ^{2}\left(\Psi-\varphi_{1}\right) \sin \left(\varphi_{3}-\varphi_{2}\right)+X_{2} \sin ^{2}\left(\Psi-\varphi_{2}\right) \sin \left(\varphi_{3}-\varphi_{1}\right)\right. \\
&\left.\quad-X_{1} \sin ^{2}\left(\Psi-\varphi_{3}\right) \sin \left(\varphi_{2}-\varphi_{1}\right)\right]
\end{aligned}
$$

This establishes the sufficiency of the conditions and the existence of the wedge $W_{\Psi}$. The uniqueness follows since the proof shows that the $r_{j}$ are uniquely determined by the 
angle of inclination $\Psi$ of the near boundary line of the wedge. It is well known that a parallel wedge cannot be reconstructed from one directed X-ray.

If we let the angle $\Psi$ approach the boundary points of the intervals in which it must lie we obtain simple sufficient conditions that three data points are X-rays of a wedge.

Corollary 3.10. If $Q(X)>0$ and

$$
X_{3} \sin \left(\varphi_{3}-\varphi_{1}\right)>X_{2} \sin \left(\varphi_{2}-\varphi_{1}\right)
$$

or

$$
X_{1} \sin \left(\varphi_{3}-\varphi_{1}\right)>X_{2} \sin \left(\varphi_{3}-\varphi_{2}\right)
$$

then the data $\left(X_{j}, \varphi_{j}\right), j=1,2,3$, are directed $X$-ray data from some wedge $W$.

Proof. If we let $\Psi<\varphi_{1}$ approach $\varphi_{1}$ in (3.7) we obtain

$$
\begin{aligned}
-X_{2} \sin ^{2}\left(\varphi_{1}-\varphi_{2}\right) \sin \left(\varphi_{3}-\varphi_{1}\right)+X_{3} \sin ^{2}\left(\varphi_{1}-\varphi_{3}\right) \sin \left(\varphi_{2}-\varphi_{1}\right) \\
=\sin \left(\varphi_{2}-\varphi_{1}\right) \sin \left(\varphi_{3}-\varphi_{1}\right)\left[-X_{2} \sin \left(\varphi_{2}-\varphi_{1}\right)+X_{3} \sin \left(\varphi_{3}-\varphi_{1}\right)\right] .
\end{aligned}
$$

Since $0<\varphi_{j}-\varphi_{i}<\pi$ when $j>i$, the last expression is positive if and only if (3.11) holds. If (3.11) holds, then (3.7) is valid for $\Psi<\varphi_{1}$ and near $\varphi_{1}$ by continuity. The sufficiency of (3.12) is established similarly by letting $\Psi>\varphi_{3}$ and approach $\varphi_{3}$.

Corollary 3.13. Suppose that $W_{1}$ and $W_{2}$ are distinct wedges having the same (nonvanishing) directed $X$-ray data along three rays $\varphi_{1}<\varphi_{2}<\varphi_{3}<\varphi_{1}+\pi$. If either the near side or the far side of $W_{1}$ is parallel to either the near side or far side of $W_{2}$, then $W_{1}$ and $W_{2}$ are parallel wedges having the same directed $X$-rays along all rays in the cone $C\left(\varphi_{1}, \varphi_{3}\right)$.

Proof. Suppose that $W_{1}$ is not a parallel wedge. Since $W_{2} \neq W_{1}$, Theorem 3.4 gives that the near sides of $W_{1}$ and $W_{2}$ cannot be parallel. Let $\omega$ be the angle of inclination of the far boundary line of $W_{1}$ and let $\left(R_{j}, \varphi_{j}\right)$ be the point of intersection of the far boundary line of $W_{1}$ with the ray $\varphi=\varphi_{j}$. Proceeding as in the proof of Theorem 3.4, one can show that

$$
R_{1}=\frac{-Q(X) \sin \left(\omega-\varphi_{2}\right) \sin \left(\omega-\varphi_{3}\right)}{T(\omega)}
$$

and that $R_{j}=\left(\sin \left(\omega-\varphi_{1}\right) / \sin \left(\omega-\varphi_{j}\right)\right) R_{1}, j=2,3$. So the intersection points with the far boundary line are uniquely determined by $\omega$ and hence the far boundary lines cannot be parallel. Since $R_{1}>0$ and $Q(X)>0$, (3.14) shows that

$$
\begin{aligned}
X_{1} \sin ^{2}\left(\omega-\varphi_{1}\right) \sin \left(\varphi_{3}-\varphi_{2}\right) & -X_{2} \sin ^{2}\left(\omega-\varphi_{2}\right) \sin \left(\varphi_{3}-\varphi_{1}\right) \\
& +X_{3} \sin ^{2}\left(\omega-\varphi_{3}\right) \sin \left(\varphi_{2}-\varphi_{1}\right)<0 .
\end{aligned}
$$


The opposite inequality, (3.7), holds for the angle of inclination of the near boundary line of a wedge. Thus a near boundary line of one of the wedges cannot be parallel to a far boundary line of the other wedge. The last statement follows from Theorem 3.1.

Remark 3.15. Note the similarity of the formulae (3.8) and (3.14) for the intersection point of the ray with the near side boundary line and far side boundary line of the wedge, respectively. If one interchanges the angles in the formulae, that is places $\omega$ in (3.8) or $\Psi$ in (3.14), then (3.8) yields $-R_{1}$ and (3.14) yields $-r_{1}$. So, one need not distinguish $\Psi$ from $\omega$ a priori. The computations of $r_{1}$ and $R_{1}$ will determine which is which. Algebraic expressions for computing the angles of inclination are given in Section 5.

Example 3.16. Do there exist wedges having X-ray data $X_{1}=4, X_{2}=6, X_{3}=5.5$ and with the angles satisfying $\varphi_{3}-\varphi_{2}=\varphi_{2}-\varphi_{1}=\pi / 18$ ? One can compute $Q(X) \sim 2.4$ and $5.5 \sin (\pi / 9)>6 \sin (\pi / 18)$. Corollary 3.10 implies that such wedges exist. We construct two such wedges when $\varphi_{1}=\pi / 9, \varphi_{2}=\pi / 6, \varphi_{3}=2 \pi / 9$. By Theorem 3.4 allowable angles of inclination for the near boundary line correspond to values of $\Psi$ not in the interval $[\pi / 9,2 \pi / 9]$ where the $\pi$-periodic function $T(\Psi)=X_{1} \sin ^{2}(\Psi-$ $\left.\varphi_{1}\right) \sin \left(\varphi_{3}-\varphi_{2}\right)-X_{2} \sin ^{2}\left(\Psi-\varphi_{2}\right) \sin \left(\varphi_{3}-\varphi_{1}\right)+X_{3} \sin ^{2}\left(\Psi-\varphi_{3}\right) \sin \left(\varphi_{2}-\varphi_{1}\right)>0$. Figure 1(a) contains a graph of $T(\Psi)$ on the intervals [ $-0.3, \pi / 9]$, [2 $\pi / 9,1] . T(\Psi)<0$ in the region of interest outside these intervals. From Fig. 1 we see that allowable values of $\Psi$ are roughly $-0.2<\Psi<0.349$ and $0.698<\Psi<0.8$. We chose $\Psi=0.2$ and $\Psi=0.735$ giving slopes of $\sim 0.203$ and $\sim 0.904$, respectively, for the near side boundary lines of the wedges. Formulae (3.8) and (3.9) were then used to construct the $r_{j}$ and hence the near side boundary line. Figure 1(b) contains the two wedges having the desired X-rays from the three chosen angles. The three rays along which the two wedges have equal directed X-rays appear as dotted lines emanating from the origin.

\section{Uniqueness from Four Rays and a Least Squares Algorithm}

In this section we prove that a nonparallel wedge is uniquely determined from its integrals along four rays. The approach taken also leads to an algorithm for reconstructing nonparallel wedges from four or more directed X-rays.

Theorem 4.1. A nonparallel wedge is uniquely determined by its integrals over four rays (that meet the wedge in segments of finite length).

First we introduce some notation and do some preliminary computations.

Consider a wedge $W$. Suppose that the cone $C(\alpha, \beta)$ intersects the wedge $W$ in the four points $r_{\alpha}, R_{\alpha}, r_{\beta}, R_{\beta}$ with $0<r_{\alpha} \leq R_{\alpha}$ the points along the ray $\varphi=\alpha$, and $0<r_{\beta} \leq R_{\beta}$ the points along the ray $\varphi=\beta$. Of course equality may hold along only one of the rays. Then for any $\varphi, \alpha \leq \varphi \leq \beta$, the points of intersection of the ray with 


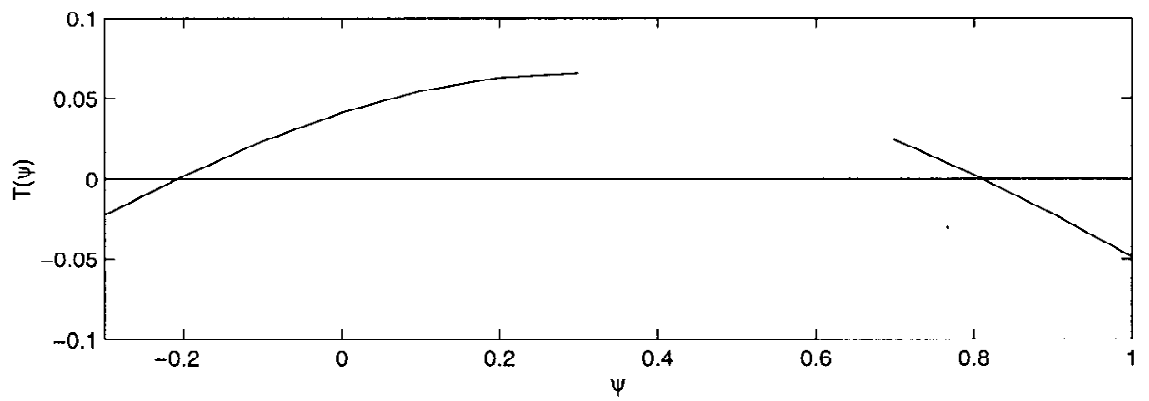

(a)

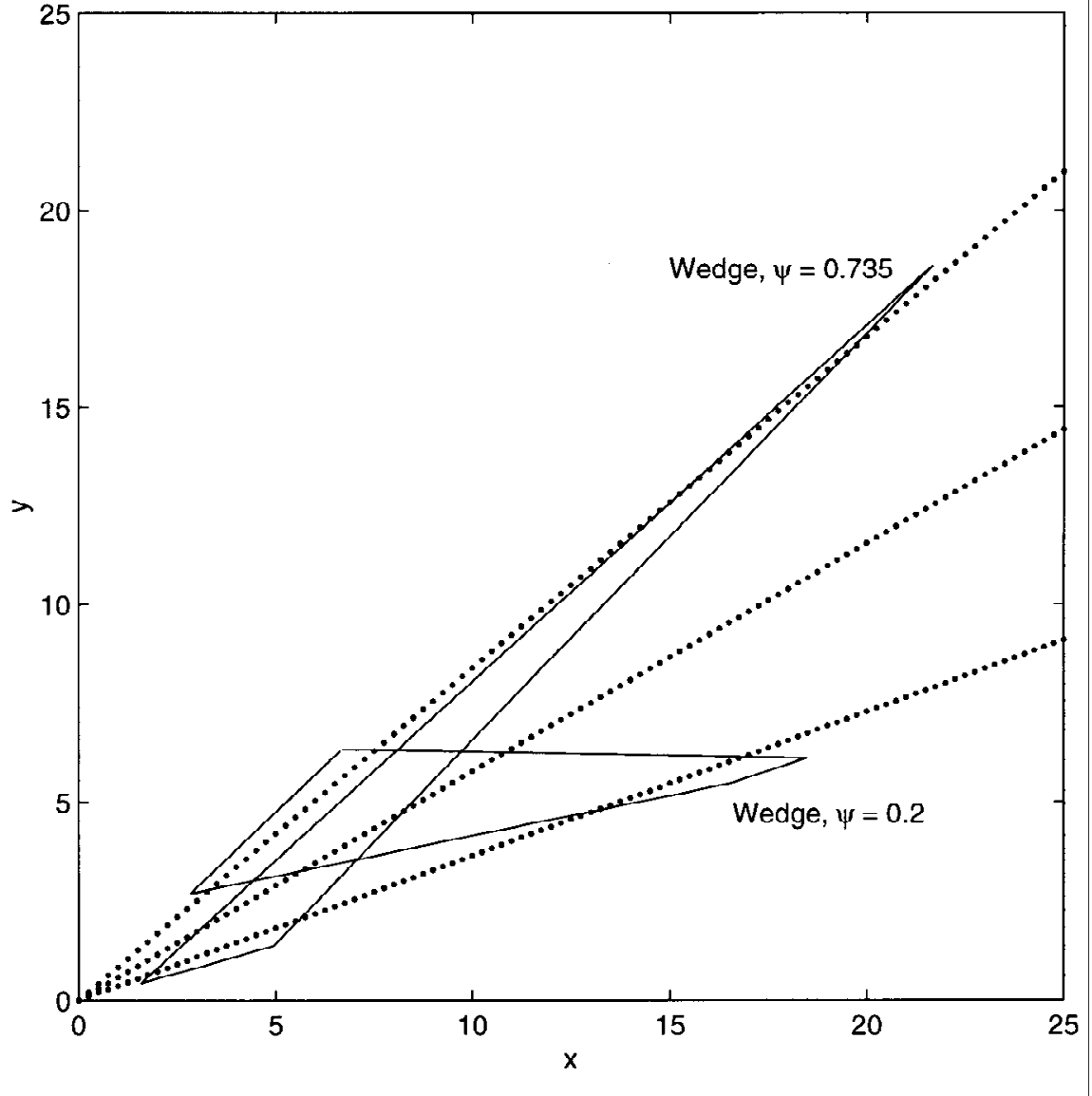

(b)

Fig. 1. (a) Graph of $T(\Psi)$. For each $\Psi$ with $T(\Psi)>0$ there is a unique wedge having the directed X-ray data of Example 3.16. (b) Wedges corresponding to $\Psi=0.2$ and $\Psi=0.735$. Dotted lines emanating from the origin (source) are the three rays over which the wedges have equal chord lengths. 
angle $\varphi$ with the near and far boundary lines of $W$ are respectively

$$
\begin{aligned}
r & =r(\varphi)=\frac{r_{\alpha} r_{\beta} \sin (\beta-\alpha)}{r_{\alpha} \sin (\varphi-\alpha)+r_{\beta} \sin (\beta-\varphi)}, \\
R & =R(\varphi)=\frac{R_{\alpha} R_{\beta} \sin (\beta-\alpha)}{R_{\alpha} \sin (\varphi-\alpha)+R_{\beta} \sin (\beta-\varphi)} .
\end{aligned}
$$

Consequently,

$$
\mathcal{D} \chi_{W}(\varphi)=\frac{R_{\alpha} R_{\beta} \sin (\beta-\alpha)}{R_{\alpha} \sin (\varphi-\alpha)+R_{\beta} \sin (\beta-\varphi)}-\frac{r_{\alpha} r_{\beta} \sin (\beta-\alpha)}{r_{\alpha} \sin (\varphi-\alpha)+r_{\beta} \sin (\beta-\varphi)} .
$$

Now suppose $W^{*}$ is another wedge with points of intersection $r_{\alpha}+a, R_{\alpha}+a, r_{\beta}+b, R_{\beta}+b$ with the rays $\varphi=\alpha$ and $\varphi=\beta$, respectively, where necessarily $a>-r_{\alpha}$ and $b>-r_{\beta}$. The wedges $W, W^{*}$ have the same directed X-rays from the angles $\alpha, \beta$. They have the same directed $\mathrm{X}$-ray from another angle $\varphi$ if and only if

$$
\begin{gathered}
\frac{R_{\alpha} R_{\beta} \sin (\beta-\alpha)}{R_{\alpha} \sin (\varphi-\alpha)+R_{\beta} \sin (\beta-\varphi)}-\frac{r_{\alpha} r_{\beta} \sin (\beta-\alpha)}{r_{\alpha} \sin (\varphi-\alpha)+r_{\beta} \sin (\beta-\varphi)} \\
=\frac{\left(R_{\alpha}+a\right)\left(R_{\beta}+b\right) \sin (\beta-\alpha)}{\left(R_{\alpha}+a\right) \sin (\varphi-\alpha)+\left(R_{\beta}+b\right) \sin (\beta-\varphi)} \\
\quad-\frac{\left(r_{\alpha}+a\right)\left(r_{\beta}+b\right) \sin (\beta-\alpha)}{\left(r_{\alpha}+a\right) \sin (\varphi-\alpha)+\left(r_{\beta}+b\right) \sin (\beta-\varphi)} .
\end{gathered}
$$

After some algebraic manipulation, we see that the last equation holds true if and only if

$$
\begin{aligned}
& {\left[R_{\alpha} r_{\beta}\left(-a r_{\beta}+2 b r_{\alpha}\right)\left(R_{\alpha}+a\right)+R_{\beta} r_{\alpha}\left(a R_{\beta}-2 b R_{\alpha}\right)\left(r_{\alpha}+a\right)\right.} \\
& \left.\quad+b^{2} R_{\alpha} r_{\alpha}\left(R_{\alpha}-r_{\alpha}\right)\right] \sin (\varphi-\alpha) \\
& \quad-\left[R_{\beta} r_{\alpha}\left(-b r_{\alpha}+2 a r_{\beta}\right)\left(R_{\beta}+b\right)\right. \\
& \left.\quad+R_{\alpha} r_{\beta}\left(b R_{\alpha}-2 a R_{\beta}\right)\left(r_{\beta}+b\right)+a^{2} R_{\beta} r_{\beta}\left(R_{\beta}-r_{\beta}\right)\right] \sin (\beta-\varphi)=0 .
\end{aligned}
$$

Our goal is to show that if $W$ and $W^{*}$ have equal X-rays along two additional directions $\varphi_{1}, \varphi_{2}$ where $\alpha<\varphi_{1}<\varphi_{2}<\beta$, then either $W=W^{*}$ or $W$ and $W^{*}$ are parallel wedges.

Lemma 4.4. If (4.3) is satisfied for two distinct values of $\varphi$ in the interval $(\alpha, \beta)$, then both

$$
\begin{aligned}
R_{\alpha} r_{\beta}\left(-a r_{\beta}+2 b r_{\alpha}\right)\left(R_{\alpha}+a\right)+R_{\beta} r_{\alpha}\left(a R_{\beta}-2 b R_{\alpha}\right)\left(r_{\alpha}+a\right) & \\
+b^{2} R_{\alpha} r_{\alpha}\left(R_{\alpha}-r_{\alpha}\right) & =0
\end{aligned}
$$

and

$$
\begin{aligned}
R_{\beta} r_{\alpha}\left(-b r_{\alpha}+2 a r_{\beta}\right)\left(R_{\beta}+b\right)+R_{\alpha} r_{\beta}\left(b R_{\alpha}-2 a R_{\beta}\right)\left(r_{\beta}+b\right) & \\
& +a^{2} R_{\beta} r_{\beta}\left(R_{\beta}-r_{\beta}\right)=0 .
\end{aligned}
$$

Proof. Formula (4.3) has the form $c_{1} \sin (\varphi-\alpha)+c_{2} \sin (\beta-\varphi)=0$ where the coefficients $c_{1}, c_{2}$ are constants. Thus the set of points $(\sin (\varphi-\alpha), \sin (\beta-\varphi))$ that 
satisfy the equation lie on a line passing through the origin. However, $\operatorname{since} \sin (\beta-\alpha)=$ $\sin (\beta-\varphi+\varphi-\alpha)=\sin (\beta-\varphi) \cos (\varphi-\alpha)+\cos (\beta-\varphi) \sin (\varphi-\alpha)$, we have that

$$
\begin{aligned}
\sin ^{2}(\beta-\alpha)= & \sin ^{2}(\beta-\varphi) \cos ^{2}(\varphi-\alpha)+2 \sin (\beta-\varphi) \cos (\varphi-\alpha) \cos (\beta-\varphi) \\
& \quad \times \sin (\varphi-\alpha)+\cos ^{2}(\beta-\varphi) \sin ^{2}(\varphi-\alpha) \\
= & \sin ^{2}(\beta-\varphi)+\sin ^{2}(\varphi-\alpha)+2 \sin (\beta-\varphi) \cos (\varphi-\alpha) \cos (\beta-\varphi) \\
& \quad \times \sin (\varphi-\alpha)-2 \sin ^{2}(\beta-\varphi) \sin ^{2}(\varphi-\alpha) \\
= & \sin ^{2}(\beta-\varphi)+\sin ^{2}(\varphi-\alpha)+2 \sin (\beta-\varphi) \sin (\varphi-\alpha) \\
& \times[\cos (\varphi-\alpha) \cos (\beta-\varphi)-\sin (\beta-\varphi) \sin (\varphi-\alpha)] \\
= & \sin ^{2}(\beta-\varphi)+\sin ^{2}(\varphi-\alpha)+2 \cos (\beta-\alpha) \sin (\beta-\varphi) \sin (\varphi-\alpha) .
\end{aligned}
$$

Hence

$$
\begin{aligned}
& \sin ^{2}(\beta-\varphi)+\sin ^{2}(\varphi-\alpha) \\
& \quad+2 \cos (\beta-\alpha) \sin (\beta-\varphi) \sin (\varphi-\alpha)=\sin ^{2}(\beta-\alpha) .
\end{aligned}
$$

Since $\alpha<\varphi<\beta$ and $\beta-\alpha<\pi$, the map $\varphi \rightarrow(\sin (\varphi-\alpha), \sin (\beta-\varphi))$ is one to one. The discriminant of (4.7) is $4 \cos ^{2}(\beta-\alpha)-4<0$, so points $(\sin (\varphi-\alpha), \sin (\beta-\varphi))$ satisfying (4.7) lie on an ellipse centered at the origin. Thus points $(\sin (\varphi-\alpha), \sin (\beta-\varphi))$ satisfying (4.3) must simultaneously lie on a line passing through the origin and an ellipse centered at the origin. There can be only one such point when $\alpha<\varphi<\beta<\alpha+\pi$ unless the coefficients $c_{1}, c_{2}$ of the line vanish identically. Hence if (4.3) vanishes for two such distinct values of $\varphi,(4.5)$ and (4.6) are satisfied.

Theorem 4.1 follows from the next lemma.

Lemma 4.8. Suppose that either $a \neq 0$ or $b \neq 0$. Equations (4.5) and (4.6) are satisfied if and only if $W$ and $W^{*}$ are parallel wedges.

Proof. Note that the left-hand side of both (4.5) and (4.6) is separately homogeneous of order 3 in the variables $\left(r_{\alpha}, R_{\alpha}, a\right)$ and $\left(r_{\beta}, R_{\beta}, b\right)$. That is, if we regard either the left-hand side of (4.5) or (4.6) as $F\left(r_{\alpha}, R_{\alpha}, a, r_{\beta}, R_{\beta}, b\right)$, then

$$
F\left(t r_{\alpha}, t R_{\alpha}, t a, r_{\beta}, R_{\beta}, b\right)=F\left(r_{\alpha}, R_{\alpha}, a, t r_{\beta}, t R_{\beta}, t b\right)=t^{3} F\left(r_{\alpha}, R_{\alpha}, a, r_{\beta}, R_{\beta}, b\right) .
$$

If $\left(r_{\alpha}, R_{\alpha}, a, r_{\beta}, R_{\beta}, b\right)$ is a solution to (4.5) and (4.6), then so is $\left(t r_{\alpha}, t R_{\alpha}, t a, s r_{\beta}, s R_{\beta}, s b\right)$ for all $t>0, s>0$. Choosing $t=1 / r_{\alpha} s=1 / r_{\beta}$ amounts to replacing $r_{\alpha}$ by 1 and $r_{\beta}$ by 1 in (4.5), (4.6). Thus (4.5), (4.6) are equivalent to

$$
\begin{aligned}
\frac{1+a}{R_{\alpha}+a} & =\frac{a R_{\alpha}-2 b R_{\alpha}-b^{2} R_{\alpha}}{a R_{\beta}^{2}-2 b R_{\alpha} R_{\beta}-b^{2} R_{\alpha}} \text { and } \\
\frac{1+b}{R_{\beta}+b} & =\frac{b R_{\beta}-2 a R_{\beta}-a^{2} R_{\beta}}{b R_{\alpha}^{2}-2 a R_{\alpha} R_{\beta}-a^{2} R_{\beta}} .
\end{aligned}
$$

Cross multiplying in these two equations gives

$$
-b^{2} R_{\alpha}-a^{2} R_{\alpha}-2 b R_{\alpha} R_{\beta}+2 b R_{\alpha}^{2}-2 a b R_{\alpha} R_{\beta}+a R_{\beta}^{2}-a R_{\alpha}^{2}+a^{2} R_{\beta}^{2}+b^{2} R_{\alpha}^{2}+2 a b R_{\alpha}=0
$$


and

$-b^{2} R_{\beta}-a^{2} R_{\beta}-2 a R_{\alpha} R_{\beta}+2 a R_{\beta}^{2}-2 a b R_{\alpha} R_{\beta}-b R_{\beta}^{2}+b R_{\alpha}^{2}+a^{2} R_{\beta}^{2}+b^{2} R_{\alpha}^{2}+2 a b R_{\beta}=0$.

Subtracting the second equation from the first gives

$$
\begin{aligned}
0= & b^{2} R_{\beta}-b^{2} R_{\alpha}+a^{2} R_{\beta}-a^{2} R_{\alpha}+2 a R_{\alpha} R_{\beta}-2 a R_{\beta}^{2}-2 b R_{\alpha} R_{\beta}+2 b R_{\alpha}^{2} \\
& -b R_{\beta}^{2}+b R_{\alpha}^{2}+2 a b R_{\alpha}-2 a b R_{\beta} \\
= & \left(R_{\beta}-R_{\alpha}\right)\left[b^{2}+a^{2}-2 a R_{\beta}-2 b R_{\alpha}+a R_{\beta}+a R_{\alpha}-b R_{\beta}+b R_{\alpha}-2 a b\right] \\
= & \left(R_{\beta}-R_{\alpha}\right)\left[(b-a)^{2}-(b-a)\left(R_{\beta}-R_{\alpha}\right)\right] \\
= & \left(R_{\beta}-R_{\alpha}\right)(b-a)\left(b-a+R_{\beta}-R_{\alpha}\right) .
\end{aligned}
$$

Thus (4.5) and (4.6) together imply that one of the following must hold:

$$
\begin{aligned}
R_{\alpha} & =R_{\beta} ; \\
a & =b ; \\
a+R_{\alpha} & =b+R_{\beta} .
\end{aligned}
$$

If $R_{\alpha}=R_{\beta}$, then the near and far sides of $W$ are parallel. Hence $W$ is a parallel wedge and since $W^{*}$ has directed X-rays in three directions in common with $W, W^{*}$ is also a parallel wedge by Theorem 3.1. If $a=b$, then the near sides of $W$ and $W^{*}$ are parallel, while if $a+R_{\alpha}=b+R_{\beta}$, then the near side of $W$ is parallel to the far side of $W^{*}$. In either case Corollary 3.13 implies that $W$ and $W^{*}$ are parallel wedges. This proves Lemma 4.8 and completes the proof of Theorem 4.1.

Corollary 4.13 [8]. With the exception of parallel wedges a convex polygon is uniquely determined by a single directed $X$-ray.

Proof. From Theorem 2.4 we can detect the finite set of rays emanating from the source on which the vertices of the polygon lie. This divides the convex polygon into a finite set of wedges, only one of which may be a parallel wedge. The nonparallel wedges are uniquely determined by Theorem 4.1. Once these have been determined, this uniquely determines the location of a remaining parallel wedge.

The proof of Theorem 4.1 leads to a system of nonlinear equations to which one can obtain a least squares solution. This algorithm may be useful in dealing with directed $\mathrm{X}$-ray data that is contaminated by noise. In the next section we give an algebraic solution to the problem of reconstructing a nonparallel wedge.

Returning to (4.2) and dividing the summands on the right-hand side by $r_{\alpha} r_{\beta}$ and $R_{\alpha} R_{\beta}$ and using the addition formula for the sine function, this equation may be rewritten in the form

$$
\begin{aligned}
\frac{\mathcal{D} \chi_{W}(\varphi)}{\sin (\beta-\alpha)}= & \frac{1}{\left(1 / R_{\beta}\right) \sin (\varphi-\alpha)+\left(1 / R_{\alpha}\right) \sin (\beta-\varphi)} \\
& -\frac{1}{\left(1 / r_{\beta}\right) \sin (\varphi-\alpha)+\left(1 / r_{\alpha}\right) \sin (\beta-\varphi)}
\end{aligned}
$$




$$
\begin{aligned}
= & \frac{1}{\left(\left(1 / R_{\alpha}\right) \sin \beta-\left(1 / R_{\beta}\right) \sin \alpha\right) \cos \varphi+\left(\left(1 / R_{\beta}\right) \cos \alpha-\left(1 / R_{\alpha}\right) \cos \beta\right) \sin \varphi} \\
& -\frac{1}{\left(\left(1 / r_{\alpha}\right) \sin \beta-\left(1 / r_{\beta}\right) \sin \alpha\right) \cos \varphi+\left(\left(1 / r_{\beta}\right) \cos \alpha-\left(1 / r_{\alpha}\right) \cos \beta\right) \sin \varphi} .
\end{aligned}
$$

Introducing the new variables

$$
\begin{array}{ll}
p=\frac{1}{r_{\alpha}} \sin \beta-\frac{1}{r_{\beta}} \sin \alpha, & q=\frac{1}{r_{\beta}} \cos \alpha-\frac{1}{r_{\alpha}} \cos \beta, \\
r=\frac{1}{R_{\alpha}} \sin \beta-\frac{1}{R_{\beta}} \sin \alpha, & s=\frac{1}{R_{\beta}} \cos \alpha-\frac{1}{R_{\alpha}} \cos \beta,
\end{array}
$$

we obtain the nonlinear equation

$$
\frac{\mathcal{D} \chi_{W}(\varphi)}{\sin (\beta-\alpha)}=\frac{1}{r \cos \varphi+s \sin \varphi}-\frac{1}{p \cos \varphi+q \sin \varphi}
$$

The nonlinear map $\left(r_{\alpha}, r_{\beta}, R_{\alpha}, R_{\beta}\right) \rightarrow(p, q, r, s)$ is a composition of the reciprocal map $\left(r_{\alpha}, r_{\beta}, R_{\alpha}, R_{\beta}\right) \rightarrow\left(1 / r_{\alpha}, 1 / r_{\beta}, 1 / R_{\alpha}, 1 / R_{\beta}\right)$ followed by a linear map whose coefficient matrix has determinant $\sin ^{2}(\beta-\alpha)>0$ since $0<\beta-\alpha<\pi$. Thus the map $\left(r_{\alpha}, r_{\beta}, R_{\alpha}, R_{\beta}\right) \rightarrow(p, q, r, s)$ is one to one and hence invertible.

An algorithm for determining $\left(r_{\alpha}, r_{\beta}, R_{\alpha}, R_{\beta}\right)$ from directed X-rays along four or more rays is now easily constructed. A simple Matlab program for the least squares algorithm is given in the Appendix.

Given $n \geq 4$ angles $\alpha=\varphi_{1}<\varphi_{2}<\cdots<\varphi_{n}=\beta$, (4.16) gives $n$ nonlinear equations in the four unknowns $p, q, r, s$. Provided initial estimates (or initial guesses) for $r_{\alpha}, r_{\beta}, R_{\alpha}, R_{\beta}$ initial estimates for $p, q, r, s$ can be obtained from (4.14) and (4.15). Beginning with these initial estimates a nonlinear least squares solution to the system of $n$ equations in four unknowns provides new values for $p, q, r, s$. Finally, one can then compute, from (4.14) and (4.15),

$$
\begin{aligned}
r_{\alpha}=\frac{\sin (\beta-\alpha)}{p \cos \alpha+q \sin \alpha}, & r_{\beta}=\frac{\sin (\beta-\alpha)}{p \cos \beta+q \sin \beta} \\
R_{\alpha}=\frac{\sin (\beta-\alpha)}{r \cos \alpha+s \sin \alpha}, & R_{\beta}=\frac{\sin (\beta-\alpha)}{r \cos \beta+s \sin \beta}
\end{aligned}
$$

Numerical experiments indicate that it is important to have reasonable initial estimates for $r_{\alpha}, r_{\beta}, R_{\alpha}, R_{\beta}$ in order that the least squares solution be accurate. In fact it was while looking for a good way to provide initial estimates for this algorithm that we found the algebraic solution derived in the next section. The results of the algebraic reconstruction can be used as initial estimates in the least squares algorithm if additional accuracy is desired.

Since $R_{\alpha}=r_{\alpha}+X_{\alpha}$ and $R_{\beta}=r_{\beta}+X_{\beta}$ where $X_{\alpha}, X_{\beta}$ are the directed line integrals along the rays $\varphi=\alpha$ and $\varphi=\beta$, two of the unknowns are easily eliminated by 
substitution. With this substitution and writing $X_{\varphi}$ in place of $\mathcal{D} \chi_{W}(\varphi)$ one obtains from (4.2) the equation

$$
\begin{aligned}
& \sin (\varphi-\alpha)\left[X_{\varphi} \sin (\varphi-\alpha)-X_{\beta} \sin (\beta-\alpha)\right] r_{\alpha}^{2} \\
& \quad+\sin (\beta-\varphi)\left[X_{\varphi} \sin (\beta-\varphi)-X_{\alpha} \sin (\beta-\alpha)\right] r_{\beta}^{2} \\
& \quad+2 X_{\varphi} \sin (\beta-\varphi) \sin (\varphi-\alpha) r_{\alpha} r_{\beta} \\
& \quad+X_{\beta} \sin (\varphi-\alpha)\left[X_{\varphi} \sin (\beta-\varphi)-X_{\alpha} \sin (\beta-\alpha)\right] r_{\alpha} \\
& \quad+X_{\alpha} \sin (\beta-\varphi)\left[X_{\varphi} \sin (\varphi-\alpha)-X_{\beta} \sin (\beta-\alpha)\right] r_{\beta}=0 .
\end{aligned}
$$

Using (1.7) one computes that the discriminant of this quadratic equation in $r_{\alpha}$ and $r_{\beta}$ is $4 \sin (\varphi-\alpha) \sin (\beta-\varphi) \sin (\beta-\alpha) Q\left(X_{\alpha}, X_{\varphi}, X_{\beta}\right)>0$. The expression is positive since $\alpha<\varphi<\beta$ and $W$ is a nonparallel wedge. So, the graph of (4.19) is a hyperbola. Given the line integrals along two rays $\varphi_{1}, \varphi_{2}$ with $\alpha<\varphi_{1}<\varphi_{2}<\beta$, one can use an implicit plotting program to graph the two hyperbolas obtained from (4.19). The unique point of intersection of the two curves in the first quadrant is the desired solution and the graphs can be used to find initial guesses for $r_{\alpha}$ and $r_{\beta}$ in the least squares algorithm. Alternatively, the graphical approximations can be used as initial guesses for a least squares solution to a system of $n \geq 2$ equations in two unknowns arising from (4.19).

\section{Algebraic Reconstruction of a Nonparallel Wedge}

In this section we give an algebraic solution to reconstructing a nonparallel wedge from integrals along four rays. We assume for simplicity that the angles $\left\{\varphi_{j}, j=1,2,3,4\right\}$ the rays make with the positive X-axis satisfy $0<\varphi_{1}<\varphi_{2}<\varphi_{3}<\varphi_{4}<\pi$. Suppose that the points of intersection of these rays with the lines forming the boundary of a nonparallel wedge $W$ are given in polar coordinates by $\left(r_{j}, \varphi_{j}\right)$ and $\left(R_{j}, \varphi_{j}\right)$. The directed X-ray data are then

$$
R_{j}-r_{j}=X_{j} \geq 0 .
$$

Since these are $\mathrm{X}$-rays of a nonparallel wedge, $X_{2}>0, X_{3}>0$, and only one of $X_{1}$ and $X_{4}$ may be zero.

Let $m=\tan \omega_{m}$ and $M=\tan \omega_{M}$ denote the slopes of the two boundary lines $L_{m}, L_{M}$ forming the wedge $W$, with $\omega_{m}, \omega_{M}$ being the angles of inclination. The points $\left(r_{j}, \varphi_{j}\right)$ lie on the near boundary line with slope $m$ and the points $\left(R_{j}, \varphi_{j}\right)$ lie on the far boundary line with slope $M$. We assume for now that neither of the lines are vertical, so $m$ and $M$ are finite. From Theorem 4.1 we know that $W$, and hence $m, M, r_{j}$, and $R_{j}$, are uniquely determined by the given data. In this section we derive algebraic formulae for these values and also derive some results which will be useful in determining when a set of data are directed X-ray data from a nonparallel wedge.

Since the data come from a nonparallel wedge, $m \neq M$ and

$$
\begin{aligned}
\tan \omega_{m} & =m=\frac{r_{j} \sin \varphi_{j}-r_{1} \sin \varphi_{1}}{r_{j} \cos \varphi_{j}-r_{1} \cos \varphi_{1}} ; \\
\tan \omega_{M} & =M=\frac{R_{j} \sin \varphi_{j}-R_{1} \sin \varphi_{1}}{R_{j} \cos \varphi_{j}-R_{1} \cos \varphi_{1}}, \quad j \neq 1 .
\end{aligned}
$$


Solving for $r_{j}$ and $R_{j}$ one obtains

$$
\begin{aligned}
r_{j} & =\frac{\sin \varphi_{1}-m \cos \varphi_{1}}{\sin \varphi_{j}-m \cos \varphi_{j}} r_{1}=\frac{\sin \left(\varphi_{1}-\omega_{m}\right)}{\sin \left(\varphi_{j}-\omega_{m}\right)} r_{1}, \quad j=2,3,4, \\
R_{j} & =\frac{\sin \varphi_{1}-M \cos \varphi_{1}}{\sin \varphi_{j}-M \cos \varphi_{j}} R_{1}=\frac{\sin \left(\varphi_{1}-\omega_{M}\right)}{\sin \left(\varphi_{j}-\omega_{M}\right)} R_{1}, \quad j=2,3,4 .
\end{aligned}
$$

The expressions for $r_{j}, R_{j}$ in (5.3), (5.4) involving the angles of inclination are valid by continuity even when $m$ or $M$ is infinite. We have proved most of the next lemma.

Lemma 5.5. If the $\left(X_{j}, \varphi_{j}\right), j=1,2,3,4,0<\varphi_{1}<\varphi_{2}<\varphi_{3}<\varphi_{4}<\pi$, are directed $X$-rays of a nonparallel wedge $W$, then the nonlinear system (in the unknowns $\left.r_{1}, R_{1}, m=\tan \omega_{m}, M=\tan \omega_{M}\right)$

$$
R_{1}-r_{1}=X_{1}, \quad c_{j} R_{1}-d_{j} r_{1}=X_{j}, \quad j=2,3,4,
$$

where

$$
c_{j}=c_{j}(M)=\frac{\sin \varphi_{1}-M \cos \varphi_{1}}{\sin \varphi_{j}-M \cos \varphi_{j}}=\frac{\sin \left(\varphi_{1}-\omega_{M}\right)}{\sin \left(\varphi_{j}-\omega_{M}\right)}, \quad j=2,3,4
$$

and

$$
d_{j}=d_{j}(m)=\frac{\sin \varphi_{1}-m \cos \varphi_{1}}{\sin \varphi_{j}-m \cos \varphi_{j}}=\frac{\sin \left(\varphi_{1}-\omega_{m}\right)}{\sin \left(\varphi_{j}-\omega_{m}\right)}, \quad j=2,3,4,
$$

has a unique solution with $r_{1}>0$ and $\omega_{m}, \omega_{M} \in\left(0, \varphi_{1}\right) \cup\left(\varphi_{4}, \pi\right)$.

Proof. We have already shown that if the data come from a nonparallel wedge, then the system (5.7), (5.8) has a solution with $r_{1}>0$. Formulae (5.3) and (5.4) show that necessarily the angles of inclination lie in one of the intervals $\left(0, \varphi_{1}\right),\left(\varphi_{4}, \pi\right)$, for, if not, one of the $r_{j}, R_{j}$ would be negative. Finally, the uniqueness of a solution with $r_{1}>0$ follows since two distinct solutions with $r_{1}>0$ lead to two distinct nonparallel wedges having the same directed X-ray data along four rays, contradicting Theorem 4.1.

The system (5.6) is linear in $r_{1}$ and $R_{1}$, but with nonlinear coefficients involving the unknowns $m=\tan \omega_{m}$ and $M=\tan \omega_{M}$. We wish to find the unique values of $m, M$ for which (5.6) has a positive solution.

Lemma 5.9. For each fixed pair of values $m \neq M$, the coefficient matrix of the linear system (5.6) has rank 2.

Proof. The coefficient matrix clearly has rank $\geq 1$. The rank is equal to 1 if and only if $c_{j}=d_{j}, j=2,3,4$. However, one readily checks that $c_{j}=d_{j}$ if and only if $(m-M) \sin \left(\varphi_{j}-\varphi_{1}\right)=0$. This can only occur when $m=M$ since $0<\varphi_{j}-\varphi_{1}<\pi$, $j \neq 1$. 
Theorem 5.10. The unique pair of values $m=\tan \omega_{m}, M=\tan \omega_{M}$ for which the system (5.6) has a solution $r_{1}>0$ is determined by the system of equations

$$
\begin{aligned}
& k_{1} m M+k_{2}(m+M)=k_{3} \\
& k_{4} m M+k_{5}(m+M)=k_{6},
\end{aligned}
$$

where

$$
\begin{aligned}
k_{1}= & -\left[\sin \left(\varphi_{3}-\varphi_{2}\right) \cos ^{2} \varphi_{1} X_{1}-\sin \left(\varphi_{3}-\varphi_{1}\right) \cos ^{2} \varphi_{2} X_{2}\right. \\
& \left.\quad+\sin \left(\varphi_{2}-\varphi_{1}\right) \cos ^{2} \varphi_{3} X_{3}\right], \\
k_{2}= & {\left[\sin \left(\varphi_{3}-\varphi_{2}\right) \sin \left(2 \varphi_{1}\right) X_{1}-\sin \left(\varphi_{3}-\varphi_{1}\right) \sin \left(2 \varphi_{2}\right) X_{2}\right.} \\
& \left.\quad+\sin \left(\varphi_{2}-\varphi_{1}\right) \sin \left(2 \varphi_{3}\right) X_{3}\right] / 2, \\
k_{3}= & \sin \left(\varphi_{3}-\varphi_{2}\right) \sin ^{2} \varphi_{1} X_{1}-\sin \left(\varphi_{3}-\varphi_{1}\right) \sin ^{2} \varphi_{2} X_{2} \\
& +\sin \left(\varphi_{2}-\varphi_{1}\right) \sin ^{2} \varphi_{3} X_{3}, \\
k_{4}= & -\left[\sin \left(\varphi_{4}-\varphi_{2}\right) \cos ^{2} \varphi_{1} X_{1}-\sin \left(\varphi_{4}-\varphi_{1}\right) \cos ^{2} \varphi_{2} X_{2}\right. \\
& \left.\quad+\sin \left(\varphi_{2}-\varphi_{1}\right) \cos ^{2} \varphi_{4} X_{4}\right], \\
k_{5}= & {\left[\sin \left(\varphi_{4}-\varphi_{2}\right) \sin \left(2 \varphi_{1}\right) X_{1}-\sin \left(\varphi_{4}-\varphi_{1}\right) \sin \left(2 \varphi_{2}\right) X_{2}\right.} \\
& \left.\quad+\sin \left(\varphi_{2}-\varphi_{1}\right) \sin \left(2 \varphi_{4}\right) X_{4}\right] / 2, \\
k_{6}= & \sin \left(\varphi_{4}-\varphi_{2}\right) \sin ^{2} \varphi_{1} X_{1}-\sin \left(\varphi_{4}-\varphi_{1}\right) \sin ^{2} \varphi_{2} X_{2} \\
& +\sin \left(\varphi_{2}-\varphi_{1}\right) \sin ^{2} \varphi_{4} X_{4} .
\end{aligned}
$$

Moreover, for $j=2,3,4$,

$$
\begin{aligned}
r_{1} & =\frac{X_{j}-c_{j} X_{1}}{c_{j}-d_{j}} \\
& =\frac{\sin \left(\varphi_{j}-\omega_{m}\right)}{\sin \left(\omega_{m}-\omega_{M}\right) \sin \left(\varphi_{j}-\varphi_{1}\right)}\left[X_{j} \sin \left(\varphi_{j}-\omega_{M}\right)-X_{1} \sin \left(\varphi_{1}-\omega_{M}\right] .\right.
\end{aligned}
$$

Proof. Assuming that we have directed X-ray data along four rays from a nonparallel wedge, we may fix $m \neq M$ so that the system (5.6) has a solution with $r_{1}>0$. With $m, M$ so fixed (5.6) is a consistent linear system of four linear equations in two unknowns. The unique solution $r_{1}$ can be obtained via Cramer's rule by pairing the equation $R_{1}-r_{1}=X_{1}$ with each of the equations $c_{j} R_{1}-d_{j} r_{1}=X_{j}, j=2,3,4$. The result is then

$$
r_{1}=\frac{X_{2}-c_{2} X_{1}}{c_{2}-d_{2}}=\frac{X_{3}-c_{3} X_{1}}{c_{3}-d_{3}}=\frac{X_{4}-c_{4} X_{1}}{c_{4}-d_{4}} .
$$

Replace the $c_{j}$ and $d_{j}$ in (5.19) by their values given in (5.7), (5.8). Rationalize the expression and apply the identity (1.13). This gives (5.18). Since $m \neq M$, and hence $c_{j} \neq d_{j}$, the equations involving $X_{1}, X_{2}, X_{3}$ and $X_{1}, X_{2}, X_{4}$ on the right-hand side of (5.19) can be rearranged to give the equivalent equations

$$
\begin{aligned}
& \left(c_{2} d_{3}-c_{3} d_{2}\right) X_{1}+\left(c_{3}-d_{3}\right) X_{2}+\left(d_{2}-c_{2}\right) X_{3}=0, \\
& \left(c_{2} d_{4}-c_{4} d_{2}\right) X_{1}+\left(c_{4}-d_{4}\right) X_{2}+\left(d_{2}-c_{2}\right) X_{4}=0 .
\end{aligned}
$$


Using the formulae for the $c_{j}, d_{j}$ in (5.7) and (5.8) one can rewrite this nonlinear system in $m$ and $M$ as the system (5.11) which is linear in $m M$ and $m+M$ with the coefficients given in (5.12)-(5.17). The details are omitted.

We show later that the system (5.11) uniquely determines the product $m M$ and the sum $m+M$. Assume that, for now, knowledge of $m M$ and $m+M$ uniquely determines $m$ and $M$ up to labeling; i.e., (5.11) determines two unique values, but without further work we cannot determine which value is $m$ and which value is $M$. Indeed one can verify this statement computationally, or recall that the problem of determining two real numbers from knowledge of their product and sum is the same as determining the eigenvalues of a $2 \times 2$ matrix from its determinant and trace. These determine the eigenvalues only up to labeling. To determine that there is a unique correct labeling of the values, it suffices to show that only one labeling leads to a positive value for $r_{1}$. Recall that from Cramer's rule

$$
r_{1}=\frac{X_{2}-c_{2} X_{1}}{c_{2}-d_{2}} \quad \text { and } \quad R_{1}=\frac{X_{2}-d_{2} X_{1}}{c_{2}-d_{2}} .
$$

From (5.7) and (5.8) interchanging the values of $m$ and $M$ interchanges $c_{2}$ and $d_{2}$ and also interchanges $r_{1}$ and $R_{1}$. So, interchanging the values of $m$ and $M$ leads to

$$
r_{1}=\frac{X_{2}-d_{2} X_{1}}{d_{2}-c_{2}} \quad \text { and } \quad R_{1}=\frac{X_{2}-c_{2} X_{1}}{d_{2}-c_{2}} .
$$

Note the value of $r_{1}$ above is the negative of the value for $R_{1}$ in (5.21). Since we know that there exists a positive solution, only one choice of labeling of $m$ and $M$ leads to a positive value for $r_{1}$. The proof of Theorem 5.10 will be complete once we show that the linear system (5.11) uniquely determines $m M$ and $m+M$. This is a tedious computation and we outline the proof.

It suffices to show that the determinant of the coefficient matrix of the system (5.11) is nonzero. Using (5.12), (5.13), (5.15), and (5.16) one sees that $k_{1} k_{5}-k_{2} k_{4}$ is a quadratic form in the X-ray data $X_{1}, X_{2}, X_{3}, X_{4}$. It thus suffices to show that the quadratic form cannot vanish for directed X-ray data $X_{1}, X_{2}, X_{3}, X_{4}$ from a nonparallel wedge. One can compute that the diagonal terms in the quadratic form all vanish. For the other terms, one obtains that the coefficient of $X_{1} X_{2}$ is

$$
\begin{aligned}
{\left[\sin \left(\varphi_{2}-\varphi_{3}\right)\right.} & \cos ^{2} \varphi_{1} \sin \left(\varphi_{1}-\varphi_{4}\right) \sin \left(2 \varphi_{2}\right) \\
+ & \left.\sin \left(\varphi_{3}-\varphi_{1}\right) \cos ^{2} \varphi_{2} \sin \left(\varphi_{4}-\varphi_{2}\right) \sin \left(2 \varphi_{1}\right)\right] / 2 \\
- & {\left[\sin \left(\varphi_{3}-\varphi_{2}\right) \sin \left(2 \varphi_{1}\right) \sin \left(\varphi_{4}-\varphi_{1}\right) \cos ^{2} \varphi_{2}\right.} \\
& \left.\quad+\sin \left(\varphi_{1}-\varphi_{3}\right) \sin \left(2 \varphi_{2}\right) \sin \left(\varphi_{2}-\varphi_{4}\right) \cos ^{2} \varphi_{1}\right] / 2 \\
= & {\left[\sin \left(\varphi_{3}-\varphi_{2}\right) \sin \left(\varphi_{4}-\varphi_{1}\right)-\sin \left(\varphi_{3}-\varphi_{1}\right) \sin \left(\varphi_{4}-\varphi_{2}\right)\right] } \\
& \times\left[\cos ^{2} \varphi_{1} \sin \left(2 \varphi_{2}\right)-\cos ^{2} \varphi_{2} \sin \left(2 \varphi_{1}\right)\right] / 2 \\
= & \cos \varphi_{1} \cos \varphi_{2} \sin \left(\varphi_{2}-\varphi_{1}\right) \\
& \times\left[\sin \left(\varphi_{3}-\varphi_{2}\right) \sin \left(\varphi_{4}-\varphi_{1}\right)-\sin \left(\varphi_{3}-\varphi_{1}\right) \sin \left(\varphi_{4}-\varphi_{2}\right)\right] \\
= & -\cos \varphi_{1} \cos \varphi_{2} \sin ^{2}\left(\varphi_{2}-\varphi_{1}\right) \sin \left(\varphi_{4}-\varphi_{3}\right),
\end{aligned}
$$


the last step following from the identity (1.13). So, the $X_{1} X_{2}$ term is

$$
-\cos \varphi_{1} \cos \varphi_{2} \sin ^{2}\left(\varphi_{2}-\varphi_{1}\right) \sin \left(\varphi_{4}-\varphi_{3}\right) X_{1} X_{2}
$$

A similar computation gives the other terms. The result is

$$
\begin{aligned}
& k_{1} k_{5}-k_{2} k_{4} \\
& =-\sin \left(\varphi_{2}-\varphi_{1}\right)\left[\cos \varphi_{1} \cos \varphi_{2} \sin \left(\varphi_{2}-\varphi_{1}\right) \sin \left(\varphi_{4}-\varphi_{3}\right) X_{1} X_{2}\right. \\
& +\cos \varphi_{2} \cos \varphi_{3} \sin \left(\varphi_{3}-\varphi_{2}\right) \sin \left(\varphi_{4}-\varphi_{1}\right) X_{2} X_{3} \\
& -\cos \varphi_{1} \cos \varphi_{3} \sin \left(\varphi_{3}-\varphi_{1}\right) \sin \left(\varphi_{4}-\varphi_{2}\right) X_{1} X_{3} \\
& +\cos \varphi_{1} \cos \varphi_{4} \sin \left(\varphi_{4}-\varphi_{1}\right) \sin \left(\varphi_{3}-\varphi_{2}\right) X_{1} X_{4} \\
& -\cos \varphi_{2} \cos \varphi_{4} \sin \left(\varphi_{4}-\varphi_{2}\right) \sin \left(\varphi_{3}-\varphi_{1}\right) X_{2} X_{4} \\
& \left.+\cos \varphi_{3} \cos \varphi_{4} \sin \left(\varphi_{4}-\varphi_{3}\right) \sin \left(\varphi_{2}-\varphi_{1}\right) X_{3} X_{4}\right] \text {. }
\end{aligned}
$$

Using Lemma 1.2 rotate the coordinates so that $\varphi_{4}=\pi / 2$. The result is then

$$
\begin{aligned}
k_{1} k_{5}-k_{2} k_{4}= & -\sin \left(\varphi_{2}-\varphi_{1}\right) \cos \varphi_{1} \cos \varphi_{2} \cos \varphi_{3} \\
& \times\left[\sin \left(\varphi_{2}-\varphi_{1}\right) X_{1} X_{2}+\sin \left(\varphi_{3}-\varphi_{2}\right) X_{2} X_{3}-\sin \left(\varphi_{3}-\varphi_{1}\right) X_{1} X_{3}\right] \\
= & -\sin \left(\varphi_{2}-\varphi_{1}\right) \cos \left(\varphi_{1}\right) \cos \left(\varphi_{2}\right) \cos \left(\varphi_{3}\right) Q\left(X_{1}, X_{2}, X_{3}\right) \neq 0 .
\end{aligned}
$$

Indeed, since $\pi / 2=\varphi_{4}>\varphi_{3}>\varphi_{2}>\varphi_{1}>-\pi / 2$ the last expression can vanish only if $Q\left(X_{1}, X_{2}, X_{3}\right)$ does. Since the data are directed X-rays of a nonparallel wedge, Lemma 1.8 and Theorem 3.1 show that $Q\left(X_{1}, X_{2}, X_{3}\right)>0$.

Remark 5.23. The assumption that $\varphi_{4}=\pi / 2$, which simplified the quadratic form, could be replaced by $\varphi_{j}=\pi / 2$. With some change in indexing the same argument as above applies.

Remark 5.24. In reconstructing a wedge from its directed X-ray data, one can first compute $m=\tan \omega_{m}$ and $M=\tan \omega_{M}$ from (5.11). (If one of these values has an exceptionally large absolute value, one can rotate the coordinates so that the value is more acceptable.) The point $r_{1}$ can then be computed by (5.18) or (3.8). Then $R_{1}=r_{1}+X_{1}$ and the remaining $r_{j}, R_{j}$ can be computed from formulae (5.3) and (5.4). (If the coordinates were rotated prior to the computation, one can rotate back to the original coordinates.) As the proof shows, and numerical computations bear out, one cannot go wrong with the choice of $m$ and $M$. If the value computed for $r_{1}$ using (5.18) or (3.8) is negative, then one has reversed $m$ and $M$. However, nothing is lost because the computed negative value is $-R_{1}$. Now one knows $R_{1}$ and the correct choice of $m$ and $M$.

It will be useful to have another equation satisfied by the values $m=\tan \omega_{m}$ and $M=\tan \omega_{M}$

Lemma 5.25. The numbers $m=\tan \omega_{m}$ and $M=\tan \omega_{M}$ satisfy the system (5.11) with coefficients in (5.12)-(5.17) if and only if they are roots of

$$
F(\mu)=\left(k_{1} k_{5}-k_{2} k_{4}\right) \mu^{2}-\left(k_{1} k_{6}-k_{3} k_{4}\right) \mu+k_{3} k_{5}-k_{2} k_{4}=0 .
$$


(Explicit formulae for the coefficients are given in (5.22) and below in (5.29) and (5.30).) Equivalently, writing $\mu=\tan \omega$, (5.26) can be written in the form

$$
\begin{aligned}
\cos ^{2} \omega & F(\tan \omega) \\
= & \sin \left(\varphi_{2}-\varphi_{1}\right) \sin \left(\varphi_{4}-\varphi_{3}\right) \sin \left(\omega-\varphi_{1}\right) \sin \left(\omega-\varphi_{2}\right) X_{1} X_{2} \\
& +\sin \left(\varphi_{3}-\varphi_{2}\right) \sin \left(\varphi_{4}-\varphi_{1}\right) \sin \left(\omega-\varphi_{2}\right) \sin \left(\omega-\varphi_{3}\right) X_{2} X_{3} \\
& -\sin \left(\varphi_{3}-\varphi_{1}\right) \sin \left(\varphi_{4}-\varphi_{2}\right) \sin \left(\omega-\varphi_{1}\right) \sin \left(\omega-\varphi_{3}\right) X_{1} X_{3} \\
& +\sin \left(\varphi_{4}-\varphi_{1}\right) \sin \left(\varphi_{3}-\varphi_{2}\right) \sin \left(\omega-\varphi_{1}\right) \sin \left(\omega-\varphi_{4}\right) X_{1} X_{4} \\
& -\sin \left(\varphi_{3}-\varphi_{1}\right) \sin \left(\varphi_{4}-\varphi_{2}\right) \sin \left(\omega-\varphi_{2}\right) \sin \left(\omega-\varphi_{4}\right) X_{2} X_{4} \\
& +\sin \left(\varphi_{2}-\varphi_{1}\right) \sin \left(\varphi_{4}-\varphi_{3}\right) \sin \left(\omega-\varphi_{3}\right) \sin \left(\omega-\varphi_{4}\right) X_{3} X_{4}=0 .
\end{aligned}
$$

Moreover $m$ or $M$ is infinite if and only if $k_{1} k_{5}-k_{2} k_{4}=0$ and if and only if $\pi / 2$ satisfies the right-hand side of (5.27).

Proof. Assuming that $k_{1} k_{5}-k_{2} k_{4} \neq 0$ Cramer's rule and (5.11) give that

$$
m+M=\frac{k_{1} k_{6}-k_{3} k_{4}}{k_{1} k_{5}-k_{2} k_{4}} \quad \text { and } \quad m M=\frac{k_{3} k_{5}-k_{2} k_{6}}{k_{1} k_{5}-k_{2} k_{4}} .
$$

It is easy then to derive that both $m$ and $M$ satisfy (5.26). The coefficient of $M^{2}$ is given in (5.22). The other terms can be computed to be

$$
\begin{aligned}
k_{3} k_{5}-k_{2} k_{6} & \\
=-\sin \left(\varphi_{2}-\varphi_{1}\right)[ & \sin \varphi_{1} \sin \varphi_{2} \sin \left(\varphi_{2}-\varphi_{1}\right) \sin \left(\varphi_{4}-\varphi_{3}\right) X_{1} X_{2} \\
& +\sin \varphi_{2} \sin \varphi_{3} \sin \left(\varphi_{3}-\varphi_{2}\right) \sin \left(\varphi_{4}-\varphi_{1}\right) X_{2} X_{3} \\
& -\sin \varphi_{1} \sin \varphi_{3} \sin \left(\varphi_{3}-\varphi_{1}\right) \sin \left(\varphi_{4}-\varphi_{2}\right) X_{1} X_{3} \\
& +\sin \varphi_{1} \sin \varphi_{4} \sin \left(\varphi_{4}-\varphi_{1}\right) \sin \left(\varphi_{3}-\varphi_{2}\right) X_{1} X_{4} \\
& -\sin \varphi_{2} \sin \varphi_{4} \sin \left(\varphi_{4}-\varphi_{2}\right) \sin \left(\varphi_{3}-\varphi_{1}\right) X_{2} X_{4} \\
& \left.+\sin \varphi_{3} \sin \varphi_{4} \sin \left(\varphi_{4}-\varphi_{3}\right) \sin \left(\varphi_{2}-\varphi_{1}\right) X_{3} X_{4}\right]
\end{aligned}
$$

and

(5.30) $k_{1} k_{6}-k_{3} k_{4}$

$$
\begin{aligned}
=-\sin \left(\varphi_{2}-\varphi_{1}\right)[ & \sin \left(\varphi_{1}+\varphi_{2}\right) \sin \left(\varphi_{2}-\varphi_{1}\right) \sin \left(\varphi_{4}-\varphi_{3}\right) X_{1} X_{2} \\
& +\sin \left(\varphi_{2}+\varphi_{3}\right) \sin \left(\varphi_{3}-\varphi_{2}\right) \sin \left(\varphi_{4}-\varphi_{1}\right) X_{2} X_{3} \\
& -\sin \left(\varphi_{1}+\varphi_{3}\right) \sin \left(\varphi_{3}-\varphi_{1}\right) \sin \left(\varphi_{4}-\varphi_{2}\right) X_{1} X_{3} \\
& +\sin \left(\varphi_{1}+\varphi_{4}\right) \sin \left(\varphi_{4}-\varphi_{1}\right) \sin \left(\varphi_{3}-\varphi_{2}\right) X_{1} X_{4} \\
& -\sin \left(\varphi_{2}+\varphi_{4}\right) \sin \left(\varphi_{4}-\varphi_{2}\right) \sin \left(\varphi_{3}-\varphi_{1}\right) X_{2} X_{4} \\
& \left.+\sin \left(\varphi_{3}+\varphi_{4}\right) \sin \left(\varphi_{4}-\varphi_{3}\right) \sin \left(\varphi_{2}-\varphi_{1}\right) X_{3} X_{4}\right] .
\end{aligned}
$$

Omitting the common factor of $-\sin \left(\varphi_{2}-\varphi_{1}\right)$, a typical term in (5.26) has the form

$$
\begin{aligned}
(-1)^{h+i+1} & \sin \left(\varphi_{i}-\varphi_{h}\right) \sin \left(\varphi_{k}-\varphi_{j}\right) X_{h} X_{i} \\
& \times\left[\mu^{2} \cos \varphi_{h} \cos \varphi_{i}-\sin \left(\varphi_{h}+\varphi_{i}\right) \mu+\sin \varphi_{h} \sin \varphi_{i}\right] \\
= & (-1)^{h+i+1} \sin \left(\varphi_{i}-\varphi_{h}\right) \sin \left(\varphi_{k}-\varphi_{j}\right)\left[\mu \cos \varphi_{h}-\sin \varphi_{h}\right]\left[\mu \cos \varphi_{i}-\sin \varphi_{i}\right],
\end{aligned}
$$


where we assume that $h, i, j, k$ take on distinct values in $\{1,2,3,4\}$ with $i>h$ and $k>j$. Writing $\mu=\tan \omega$ and multiplying through by $\cos ^{2} \omega$ this last expression becomes

$$
(-1)^{h+i+1} \sin \left(\varphi_{i}-\varphi_{h}\right) \sin \left(\varphi_{k}-\varphi_{j}\right) \sin \left(\omega-\varphi_{h}\right) \sin \left(\omega-\varphi_{i}\right) .
$$

This establishes (5.27). Finally, $m$ or $M$ being infinite is equivalent to $\omega=\pi / 2$, and from (5.22) the right-hand side of (5.27) vanishes when $\omega=\pi / 2$ if and only if $k_{1} k_{5}-k_{2} k_{4}$ $=0$.

We now give a partial converse to Lemma 5.5 which is helpful in characterizing directed X-ray data from a nonparallel wedge.

Lemma 5.31. Let $\left(X_{j}, \varphi_{j}\right), j=1,2,3,4$, be given with $0<\varphi_{1}<\varphi_{2}<\varphi_{3}<\varphi_{4}<\pi$, each $X_{j} \geq 0, X_{2}>0, X_{3}>0$, and at most one of $X_{1}, X_{4}$ equal to 0 . If the system (5.6) (with $c_{j}, d_{j}$ defined by (5.7), (5.8)) has a solution with $r_{1}>0$ and the angles $\omega_{m} \neq \omega_{M}$ lying in the set $\left(0, \varphi_{1}\right) \cup\left(\varphi_{4}, \pi\right)$, then there exists a unique nonparallel wedge $W$ such that $\mathcal{D} \chi_{W}\left(\varphi_{j}\right)=X_{j}, j=1,2,3,4$.

Proof. Suppose that $r_{1}>0, R_{1}, \omega_{m} \neq \omega_{M}$ are solutions to (5.6) with the angles lying in the required range. Then $R_{1}=r_{1}+X_{1}>0$. Define $r_{j}, R_{j}, j=2,3,4$, by (5.3) and (5.4). By the assumption on the range of the angles these are positive. By construction the points satisfy $R_{j}-r_{j}=X_{j}$ and the points $\left(r_{j}, \varphi_{j}\right)$ are collinear and so are the points $\left(R_{j}, \varphi_{j}\right)$. Thus the data come from a nonparallel wedge. The uniqueness is a consequence of Theorem 4.1.

Remark. The rotation invariance of the reconstruction problem is evident in the formula (5.18). Indeed, this formula is invariant under translations of all the angles including $\omega_{m}$ and $\omega_{M}$. That is, if $\varphi_{j}$ is replaced by $\varphi_{j}+\alpha, j=1,2,3$, and $\omega_{m}$ and $\omega_{M}$ are replaced by $\omega_{m}+\alpha$ and $\omega_{M}+\alpha$, then the value computed remains the same. With some elementary but lengthy algebra we have eliminated the variables $\omega_{m}$ and $\omega_{M}$ from (5.18). This gives a formula for $r_{1}$ that directly involves only the directed $\mathrm{X}$-ray data $\left(X_{j}, \varphi_{j}\right)$. This formula is invariant under rotations of the angles $\varphi_{j}$. Indeed, these angles only occur in expressions of the form $\sin \left(\varphi_{j}-\varphi_{k}\right)$. This rotation invariant formula is extremely long and has not been useful in reconstructing wedges. It gave reasonable reconstructions when the angle between consecutive rays was about $5^{\circ}$, but due to loss of significance errors gave poor results for smaller spacing. Therefore, we only mention the existence of such a formula, but omit giving it explicitly.

\section{Characterization of Discrete Data for a Nonparallel Wedge}

In this section we give necessary and sufficient conditions that a discrete set $\left\{\left(X_{j}, \varphi_{j}\right)\right.$, $j=1, \ldots, n\}$ be directed X-ray data for a nonparallel wedge. We assume throughout this section that the data satisfy the following condition. 
Condition N. The discrete data set $\left\{\left(X_{j}, \varphi_{j}\right), j=1, \ldots, n\right\}$ satisfies $0<\varphi_{1}<\cdots<$ $\varphi_{n}<\pi$, with each $X_{j}>0$ with the possible exception of $X_{1}$ or $X_{n}$, one of which may be zero. Further, for $j=1,2, \ldots, n-2, Q\left(X_{j}, X_{j+1}, X_{j+2}\right)>0$.

Given the ordering of the angles, the conditions on the $X_{j}$ are obviously necessary. Lemma 1.12 and the remark following explain the last statement in Condition $\mathrm{N}$. We assume that $0<\varphi_{1}<\cdots<\varphi_{n}<\pi$ for convenience to ensure that the data come from a body in the upper half-plane. We first treat the case of directed X-ray data along four rays.

Theorem 6.1. Suppose that $\left(X_{j}, \varphi_{j}\right), j=1,2,3,4$, satisfy Condition N. Then there exists a unique nonparallel wedge $W$ that does not contain the origin and such that $\mathcal{D} \chi_{W}\left(\varphi_{j}\right)=X_{j}, j=1,2,3$, 4 , if and only if the following conditions are satisfied:

$$
\left(k_{1} k_{6}-k_{3} k_{4}\right)^{2}-4\left(k_{1} k_{5}-k_{2} k_{4}\right)\left(k_{3} k_{5}-k_{2} k_{6}\right)>0,
$$

where $k_{1} \ldots, k_{6}$ are given in (5.12)-(5.17); and

$$
\omega_{m} \text { or } \omega_{M} \in\left(0, \varphi_{1}\right) \cup\left(\varphi_{4}, \pi\right),
$$

where $m=\tan \omega_{m}$ and $M=\tan \omega_{M}$ are the solutions of (5.28).

Proof. Suppose there exists a nonparallel wedge $W$ such that $\mathcal{D} \chi_{W}\left(\varphi_{j}\right)=X_{j}, j=$ $1,2,3,4$. If the slopes $m \neq M$ of the boundary lines of the nonparallel wedge are finite they satisfy the quadratic equation (5.28). If one of the slopes is infinite, then $k_{1} k_{5}-k_{2} k_{4}=0$ and the finite slope still satisfies (5.28). In either case inequality (6.2) holds. The angles of rays that meet a wedge in an interval of finite length cannot be parallel to either the near or far boundary line of the wedge. So, $\omega_{m} \neq \varphi_{j}$ for all $j$. Since all the $r_{j}$ are positive, (5.3) shows that (6.3) is satisfied. Indeed, $r_{1}>0$ and if $\varphi_{j-1}<\omega_{m}<\varphi_{j}$, then (5.3) implies that $r_{j}<0$.

We now prove the sufficiency. If (6.2) is satisfied, then we may compute distinct solutions $m=\tan \omega_{m}$ and $M=\tan \omega_{M}$ of (5.26), equivalently (5.27). (If $k_{1} k_{5}-k_{2} k_{4}=0$, Lemma 5.25 shows that one of the two values $m, M$ is infinite and we may take the corresponding angle of inclination to be $\pi / 2$. Alternatively, we may rotate the coordinates so that $k_{1} k_{5}-k_{2} k_{4} \neq 0$ and $m$ and $M$ are both finite.) With the aid of (1.7) and Lemma 1.12, we see that the function $F$ of (5.26), (5.27) satisfies

$$
\begin{aligned}
& \cos ^{2} \varphi_{1} F\left(\tan \varphi_{1}\right)=\sin \left(\varphi_{2}-\varphi_{1}\right) \sin \left(\varphi_{3}-\varphi_{1}\right) \sin \left(\varphi_{4}-\varphi_{1}\right) Q\left(X_{2}, X_{3}, X_{4}\right)>0, \\
& \cos ^{2} \varphi_{2} F\left(\tan \varphi_{2}\right)=\sin \left(\varphi_{2}-\varphi_{1}\right) \sin \left(\varphi_{3}-\varphi_{2}\right) \sin \left(\varphi_{4}-\varphi_{2}\right) Q\left(X_{1}, X_{3}, X_{4}\right)>0, \\
& \cos ^{2} \varphi_{3} F\left(\tan \varphi_{3}\right)=\sin \left(\varphi_{3}-\varphi_{1}\right) \sin \left(\varphi_{3}-\varphi_{2}\right) \sin \left(\varphi_{4}-\varphi_{3}\right) Q\left(X_{1}, X_{2}, X_{4}\right)>0, \\
& \cos ^{2} \varphi_{4} F\left(\tan \varphi_{4}\right)=\sin \left(\varphi_{4}-\varphi_{1}\right) \sin \left(\varphi_{4}-\varphi_{2}\right) \sin \left(\varphi_{4}-\varphi_{3}\right) Q\left(X_{1}, X_{2}, X_{3}\right)>0 .
\end{aligned}
$$

Hence, $\varphi_{j}$ cannot be a root of $F$ and the number of roots of $F$ between any two consecutive values of the data angles must be even. Since there are only two roots of $F$ in any interval of length $\pi$ and by $(6.3)$ one of them lies in the set $\left(0, \varphi_{1}\right) \cup\left(\varphi_{4}, \pi\right)$, the other does also. So both $\omega_{m}$ and $\omega_{M} \in\left(0, \varphi_{1}\right) \cup\left(\varphi_{4}, \pi\right)$. 
Now reverse the derivation of the algebraic solution of reconstructing a wedge in the previous section. Since $m \neq M$ the constants $c_{j}, d_{j}$ in (5.7), (5.8) are distinct. Moreover, $m$ and $M$ are the unique solutions to (5.11) where the coefficients are given by (5.12)(5.17). Thus $m$ and $M$ also satisfy (5.20), and hence equivalently the equations in (5.19) involving $X_{1}, X_{2}, X_{3}$ and $X_{1}, X_{2}, X_{4}$ are satisfied. That is,

$$
\frac{X_{2}-c_{2} X_{1}}{c_{2}-d_{2}}=\frac{X_{3}-c_{3} X_{1}}{c_{3}-d_{3}}=\frac{X_{4}-c_{4} X_{1}}{c_{4}-d_{4}} .
$$

The common value in (6.4) is nonzero. Indeed, if it is zero (6.4) and (5.7) imply that the points $\left(X_{j}, \varphi_{j}\right)$ are collinear. From Lemma 1.5(a), $Q\left(X_{1}, X_{2}, X_{3}\right)=Q\left(X_{2}, X_{3}, X_{4}\right)=$ 0 , contradicting Condition N. Suppose the value in (6.4) is positive. Then we may use (6.4) to define $r_{1}$. Now set $R_{1}=r_{1}+X_{1}$. Then, for $j=2,3,4, c_{j} R_{1}-d_{j} r_{1}=$ $\left(c_{j}-d_{j}\right) r_{1}+c_{j} X_{1}=X_{j}-c_{j} X_{1}+c_{j} X_{1}=X_{j}$. By Lemma 5.31, the data is directed $X$-ray data of a nonparallel wedge. If the value in (6.4) is negative, then we reverse $m$ and $M$. This reverses the sign of the expressions in (6.4) and the previous argument can be applied.

Example 6.5. Condition $\mathrm{N}$ and (6.2) do not imply (6.3). Indeed, consider the directed $\mathrm{X}$-ray data $\left(X_{j}, \varphi_{j}\right), j=1,2,3,4$,

$$
\left(\frac{1}{2}, 0\right),\left(1.2958, \arctan \left(\frac{1}{4}\right)\right),\left(5.2175, \arctan \left(\frac{1}{2}\right)\right),(1, \pi / 2) .
$$

The value computed from (6.2) is $\sim 0.0165$, while $Q\left(X_{1}, X_{2}, X_{3}\right) \sim 0.4571$ and $Q\left(X_{2}, X_{3}, X_{4}\right) \sim 4.8762$. These lead to values of $\omega_{m} \sim 0.5880$ and $\omega_{M} \sim 1.1071$. So, $\omega_{m}$ and $\omega_{M}$ lie between $\varphi_{3}=\arctan \left(\frac{1}{2}\right) \sim 0.4636$ and $\varphi_{4}=\pi / 2$. Hence (6.3) is not satisfied. With the computed values of $\omega_{m}$ and $\omega_{M}$ formulae (5.18), (5.3), and (5.4) give negative values for $r_{4}$ and $R_{4}$. The four reconstructed points $\left(r_{j}, \varphi_{j}\right)$ are collinear as are the four points $\left(R_{j}, \varphi_{j}\right)$. They also satisfy $R_{j}-r_{j}=X_{j}, j=1,2,3,4$. However, the "near" and "far" side lines cross and form two wedges. Figure 2 shows the lines determined by the $r_{j}$ and $R_{j}$. Dotted lines emanating from the origin represent the rays of integration. The distance from the origin to an asterisk $(*)$ on a ray indicates the directed $\mathrm{X}$-ray datum for that ray.

It is interesting that even though a nonparallel wedge is uniquely determined by its integrals along four rays, the necessary and sufficient conditions that four data points be directed X-ray data of some nonparallel wedge $W$ is an open condition. So, small perturbations of four data points from a wedge $W$ are directed X-ray data for some other wedge $W^{*}$. We initially had the condition (6.8) below as a hypothesis in Theorem 6.1, but noticed while doing numerical experiments that whenever (6.2) and (6.3) were satisfied, then so was (6.8).

The fact that the consistency condition for wedges and four angles is an open condition suggests (and as we shall see later examples confirm) that the integrals over four rays from a section of a polygon containing a vertex may also be directed X-ray data of a nonparallel wedge. For this reason it is useful to have a way to check when more than four points are directed X-ray data from the same nonparallel wedge.

Theorem 6.6. Suppose that a set of data points $\left\{\left(X_{p}, \varphi_{p}\right), p=1,2, \ldots, n\right\}$ is given that satisfy Condition N. Suppose further that it is known that four of the data points, 


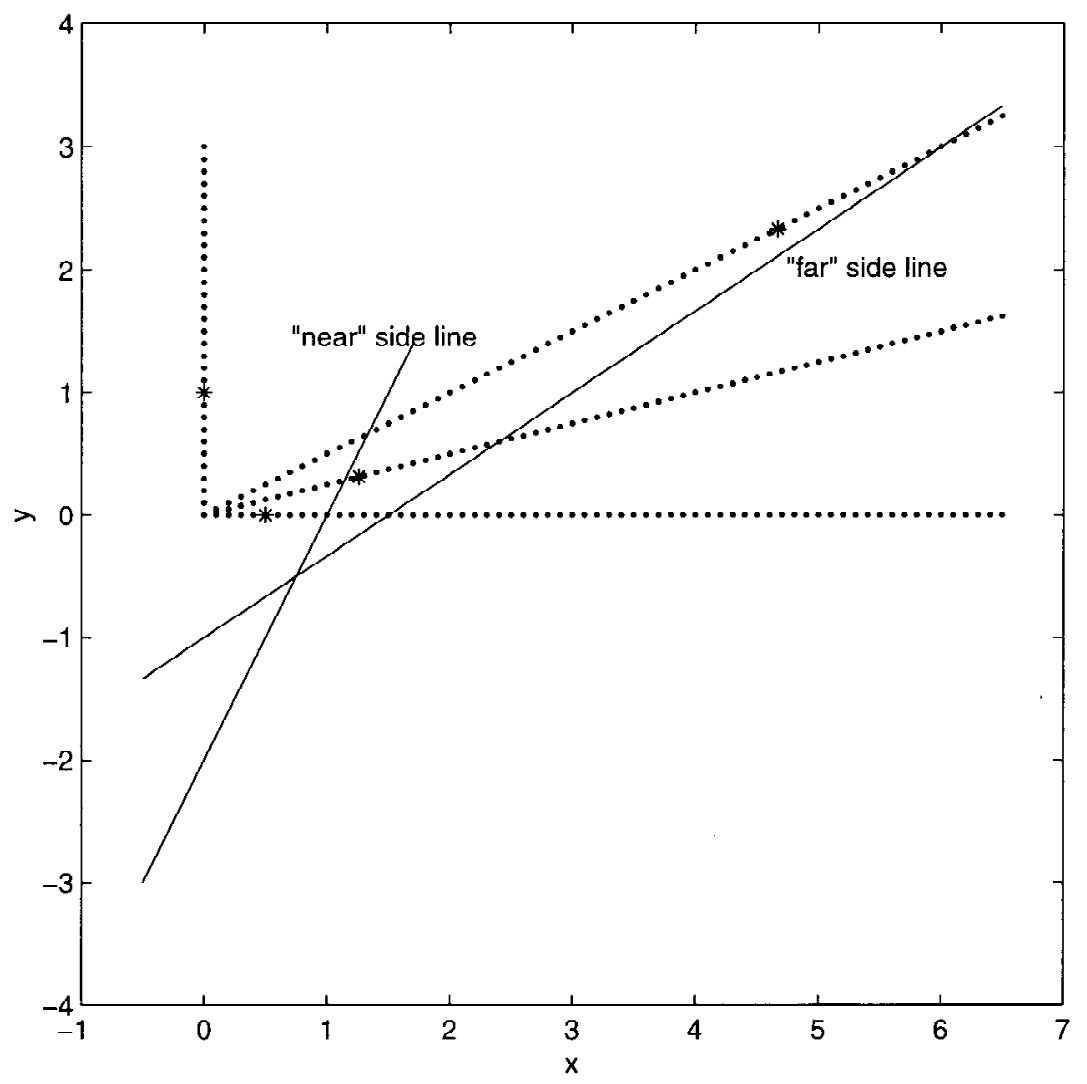

Fig. 2. Four dotted lines emanating from the origin give the rays of integration. X-ray data (*) satisfying Condition $\mathrm{N}$ and (6.2), but not (6.3), lead to reconstruction of two crossing lines instead of a wedge.

say $X_{h}, X_{i}, X_{j}, X_{k}$ with $1 \leq h<i<j<k \leq n$, are directed $X$-ray data from a nonparallel wedge $W$. Then $\mathcal{D} \chi_{W}\left(\varphi_{p}\right)=X_{p}, p=1,2, \ldots, n$, if and only if

$$
\omega_{m}, \omega_{M} \in\left(0, \varphi_{1}\right) \cup\left(\varphi_{n}, \pi\right),
$$

and

$$
\begin{aligned}
& X_{h} \sin \left(\varphi_{h}-\omega_{M}\right) \sin \left(\varphi_{h}-\omega_{m}\right) \sin \left(\varphi_{i}-\varphi_{p}\right)+X_{i} \sin \left(\varphi_{i}-\omega_{M}\right) \\
& \quad \times \sin \left(\varphi_{i}-\omega_{m}\right) \sin \left(\varphi_{p}-\varphi_{h}\right)-X_{p} \sin \left(\varphi_{p}-\omega_{M}\right) \sin \left(\varphi_{p}-\omega_{m}\right) \\
& \quad \times \sin \left(\varphi_{i}-\varphi_{h}\right)=0 \quad \text { for } \quad 1 \leq p \leq n, \quad p \neq h, i, j, k,
\end{aligned}
$$

where $\omega_{m}$ and $\omega_{M}$ are the slopes of the near and far boundary lines of the wedge $W$. (Either or both of the subscripts $h, i$ may be replaced by $j$ or $k$ throughout.) 
Proof. The necessity of (6.7) is clear. To see the necessity of (6.8), note that if $\mathcal{D} \chi_{W}\left(\varphi_{p}\right)=$ $X_{p}$, then we have, from (5.19),

$$
\frac{X_{p}-c_{p} X_{h}}{c_{p}-d_{p}}=\frac{X_{i}-c_{i} X_{h}}{c_{i}-d_{i}}, \quad p \neq h, i
$$

where $c_{j}, d_{j}$ are defined in (5.7), (5.8). The left-hand side of (6.9) is (5.18) with 1 replaced by $h$ and $j$ replaced by $p$, while the right-hand side of (6.9) is (5.18) with 1 replaced by $h$ and $j$ replaced by $i$. Thus (6.9) gives

$$
\begin{aligned}
& \frac{\sin \left(\varphi_{p}-\omega_{m}\right)}{\sin \left(\varphi_{p}-\varphi_{h}\right)}\left[X_{p} \sin \left(\varphi_{p}-\omega_{M}\right)-X_{h} \sin \left(\varphi_{h}-\omega_{M}\right)\right] \\
& =\frac{\sin \left(\varphi_{i}-\omega_{m}\right)}{\sin \left(\varphi_{i}-\varphi_{h}\right)}\left[X_{i} \sin \left(\varphi_{i}-\omega_{M}\right)-X_{h} \sin \left(\varphi_{h}-\omega_{M}\right)\right] .
\end{aligned}
$$

Rationalizing this expression and using the trigonometric identities (1.13) and (1.14) several times leads to (6.8). Thus the necessity is established.

The sufficiency of the conditions is now easy. Fix $p$ so that $p \neq h, i, j$, or $k$. Since the data $\left(X_{h}, \varphi_{h}\right),\left(X_{i}, \varphi_{i}\right),\left(X_{j}, \varphi_{j}\right),\left(X_{k}, \varphi_{k}\right)$ come from a wedge $W$, the system (5.6) has a unique positive solution $r_{1}$ when $X_{1}$ is replaced by $X_{h}, X_{2}$ by $X_{i}$, etc. Condition $\mathrm{N}$ ensures that the data lie on a convex body with the origin as a boundary point, and in light of Theorem 6.1 condition (6.7) ensures that the angles of inclination of the wedge are consistent with the given ray angles. Finally, reversing the derivation of (6.8) we see that (6.8) implies that (6.9) holds. By (5.19), (6.9) then holds when $p$ is replaced by $i, j$, or $k$. Thus the system (5.6) also has the solution $r_{1}$ when any of $h, i, j, k$ is replaced by $p$. By Lemma 5.31, D $\chi_{W}\left(\varphi_{p}\right)=X_{p}$.

Remark 6.10. The compatibility condition (6.8) was derived from the reconstruction formula (5.18). Following the same procedure as above, but using reconstruction formula (3.8) in place of (5.18), one can obtain a compatibility condition that can be used in place of (6.8). Indeed, if $h<i<p$ and $p \neq j$ or $k$, one can use (3.8) to compute $r_{h}$ in two ways, first using data $\omega_{m}, X_{h}, X_{i}, X_{j}$ and then using $\omega_{m}, X_{h}, X_{i}, X_{p}$. Equating the two expressions for $r_{h}$ one obtains the condition

$$
\begin{aligned}
& Q\left(X_{h},\right.\left.X_{i}, X_{j}\right) \sin \left(\omega_{m}-\varphi_{j}\right)\left[X_{h} \sin ^{2}\left(\omega_{m}-\varphi_{h}\right) \sin \left(\varphi_{j}-\varphi_{i}\right)-X_{i} \sin ^{2}\left(\omega_{m}-\varphi_{i}\right)\right. \\
&\left.\quad \times \sin \left(\varphi_{p}-\varphi_{h}\right)+X_{j} \sin ^{2}\left(\omega_{m}-\varphi_{j}\right) \sin \left(\varphi_{i}-\varphi_{h}\right)\right] \\
&=Q\left(X_{h}, X_{i}, X_{p}\right) \sin \left(\omega_{m}-\varphi_{p}\right) \\
& \quad \times\left[X_{h} \sin ^{2}\left(\omega_{m}-\varphi_{h}\right) \sin \left(\varphi_{p}-\varphi_{i}\right)-X_{i}\right. \\
&\left.\quad \times \sin ^{2}\left(\omega_{m}-\varphi_{i}\right) \sin \left(\varphi_{p}-\varphi_{h}\right)+X_{p} \sin ^{2}\left(\omega_{m}-\varphi_{p}\right) \sin \left(\varphi_{i}-\varphi_{h}\right)\right] .
\end{aligned}
$$

\section{Reconstructions}

For all reconstructions discrete directed $\mathrm{X}$-ray data were used with the rays equally spaced with angle $\Delta>0$ between consecutive rays. The choice of $\Delta$ was different for different reconstructions. Ideally one wants to choose $\Delta$ so that at least four rays, preferably five, pass through each wedge in the polygon, but this requires a priori information about the polygon and its location. Each polygon was reconstructed as follows. 
Theorem 2.4 and formula (2.5) were used to locate cones with vertex angle $\Delta$ that contain rays upon which vertices of the polygon lie. If $\Delta$ is sufficiently small, then each of the cones contains exactly one ray that passes through a vertex. This determines a finite collection of cones $\left\{C_{j}\right\}$, each of which intersects the polygon in a distinct wedge $W_{j}$. Next, the values $Q\left(X_{j}, X_{j+1}, X_{j+2}\right)$ were computed and Theorem 3.1 used to determine whether any of the $W_{j}$ is a parallel wedge. (Since a convex polygon can contain at most one parallel wedge, the nonparallel wedges were reconstructed. After that, the location of any parallel wedge is clear.) For each of the nonparallel wedges $W_{j}$, Theorem 6.1 and (6.8) (or (6.11)) was used to confirm that the directed X-ray data from the cone $C_{j}$ was consistent with being directed X-ray data from a nonparallel wedge. The slopes of the near and far boundary lines of the wedge were computed from the system (5.11) and the points of intersection of the rays with the wedge were computed using either (3.8) and (3.9) or (5.18) and (5.3).

The reconstructions were all done using Matlab and "exact" (as computed by Matlab) data. In numerical computations Matlab carries fifteen decimal digits and in "format short" displays five decimal places. In reconstructing wedges the computed points of intersection, slopes, and angles of inclination all agreed with the "exact" values to all places shown in format short. Occasionally, we looked at the results in "format long", fifteen decimal places, and the computed and true results agreed to ten or more places. It seems that four to five significant digits were lost when computing with $\Delta=0.005$ radians $\sim 0.29^{\circ}$ between consecutive rays.

Example 7.1. First we consider the simple pentagon bounded by the lines $y+3 x=$ $30,4 y+3 x=48,5 y+3 x=30$, and the rays $\varphi=28.5^{\circ} \sim 0.4974$ radians and $\varphi=48.5^{\circ} \sim 0.8465$ radians. The first two lines determine the far boundary. There is a vertex at $(8,6)$ along the ray $\varphi=\arctan \left(\frac{3}{4}\right) \sim 0.6435$. The near boundary consists of the third line.

Directed X-ray data were taken over the cone $C\left(28.5^{\circ}, 48.5^{\circ}\right)$ with the first ray at $28.5^{\circ}$ and $\Delta=2.5^{\circ} \sim 0.0436$ radians. So, data were taken from nine equally spaced rays in the cone. A graph of a first-order divided difference approximation to $X_{-}^{\prime}\left(\varphi_{0}\right)-X_{+}^{\prime}\left(\varphi_{0}\right)=$ $\left(\mathcal{D} \chi_{K}\right)_{-}^{\prime}\left(\varphi_{0}\right)-\left(\mathcal{D} \chi_{K}\right)_{+}^{\prime}\left(\varphi_{0}\right)$ for rays $2-8$ is shown in Fig. 3(a). This graph indicates that there is a vertex between the rays $\varphi=0.6$ and $\varphi=0.7$, that is, between the fourth and fifth rays.

Theorem 6.1 was applied to each of the six sets of four consecutive data points. Each of the six sets satisfied condition (6.2), and all the sets with the exception of the second and fourth satisfied (6.3). So the sets $\left\{\left(X_{j}, \varphi_{j}\right), j=1,2,3,4\right\},\left\{\left(X_{j}, \varphi_{j}\right)\right.$, $j=3,4,5,6\},\left\{\left(X_{j}, \varphi_{j}\right), j=5,6,7,8\right\}$, and $\left\{\left(X_{j}, \varphi_{j}\right), j=6,7,8,9\right\}$ are each directed $\mathrm{X}$-ray data of a unique nonparallel wedge.

The data set $\left\{\left(X_{j}, \varphi_{j}\right), j=3,4,5,6\right\}$ is consistent with coming from both Polygon 1 and a distinct nonparallel wedge $W^{*}$. The near and far boundary lines of $W^{*}$ were reconstructed. Both of these lines and Polygon 1 appear in Fig. 4. The dotted rays emanating from the origin are the four rays over which the wedge and polygon have equal integrals. The fact that Polygon 1 and the wedge have the same integrals along four rays can also be seen in Fig. 3(b). There, graphs of $X(\varphi)$, the directed X-ray data for both the wedge (dashed curve) and polygon, appear. The four intersection points of the graphs determine the four angles at which the wedge and polygon section have equal directed X-rays. 


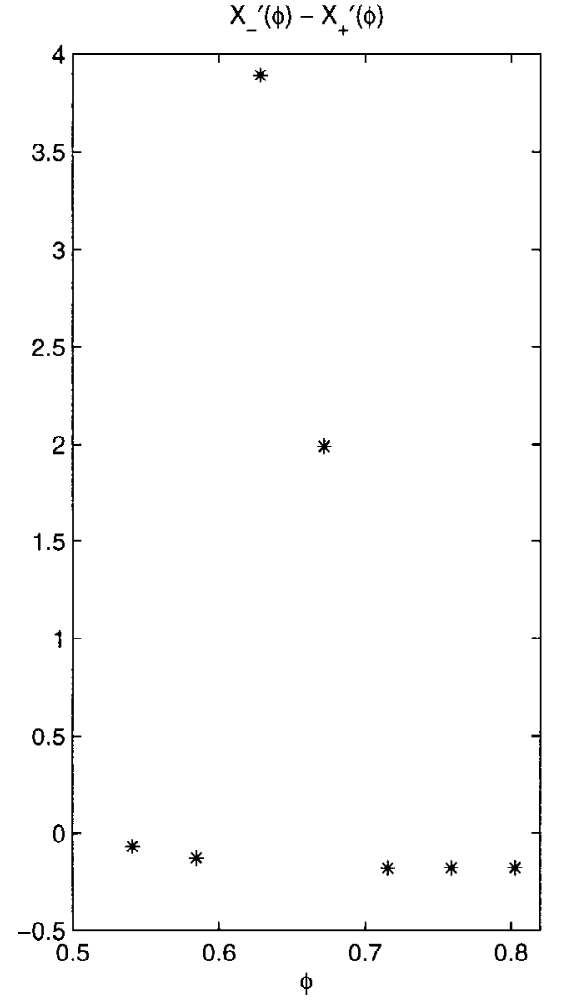

(a)

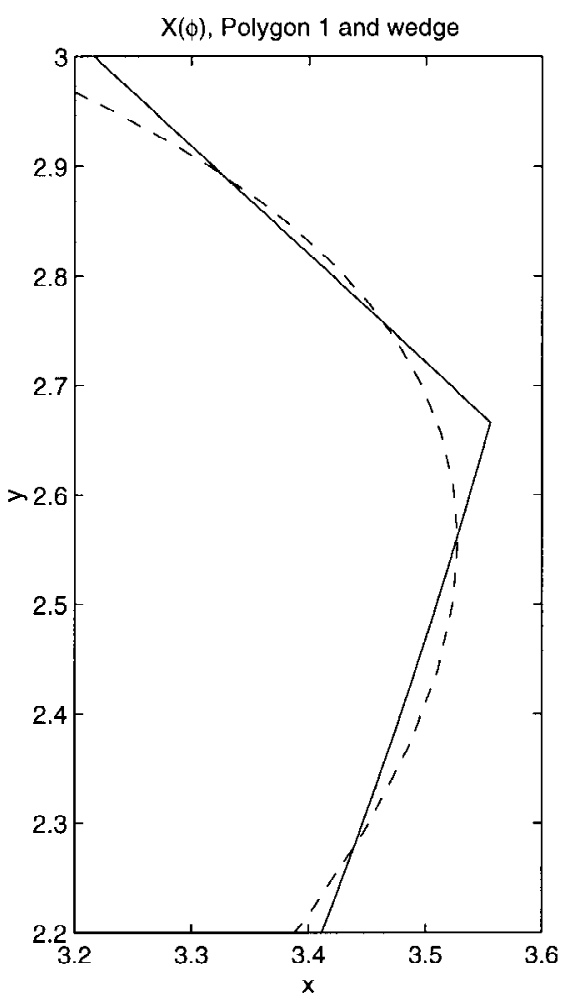

(b)

Fig. 3. (a) Divided difference approximation to difference in left and right derivatives of directed X-ray for Polygon 1. Jumps indicate approximate location of vertices. (b) Graphs of the directed X-ray of a wedge (dashed line) and a section of Polygon 1 containing a vertex (solid line). The crossing points determine the four rays for which the integrals are equal.

Theorem 6.6 settled any confusion by showing that the last five data points were directed X-ray data of a nonparallel wedge that was distinct from the wedge determined by the first four data points. The polygon, its directed X-ray data $(x)$, and the points reconstructed from the data $(+)$ appear in Fig. 5.

For amusement we also took directed X-rays spaced at $1^{\circ}$ intervals that passed through the vertex at $(8,6)$, and compared the right-hand side of $(2.5)$ (which turned out to be 6.2500) with the divided differences approximation to the left-hand side of (2.5). The latter was 6.1711 .

Example 7.2. Polygon 2 is a hexagon with vertices $(8,8),(5,5),(4,12),(-4,12)$, $(-2,4)$, and $(-8,7)$. The far right edge of the polygon lies along a ray from the source while rays from the source meet the far left edge in a vertex. Polygon 2 lies in the cone $0.7854 \sim \pi / 4 \leq \varphi \leq \pi-\arctan \left(\frac{7}{8}\right) \sim 2.423$. The vertices between supporting rays occur along the rays $\varphi \sim 1.249, \varphi \sim 1.8925$, and $\varphi \sim 2.0344$. Here $\Delta=0.02$ radians 


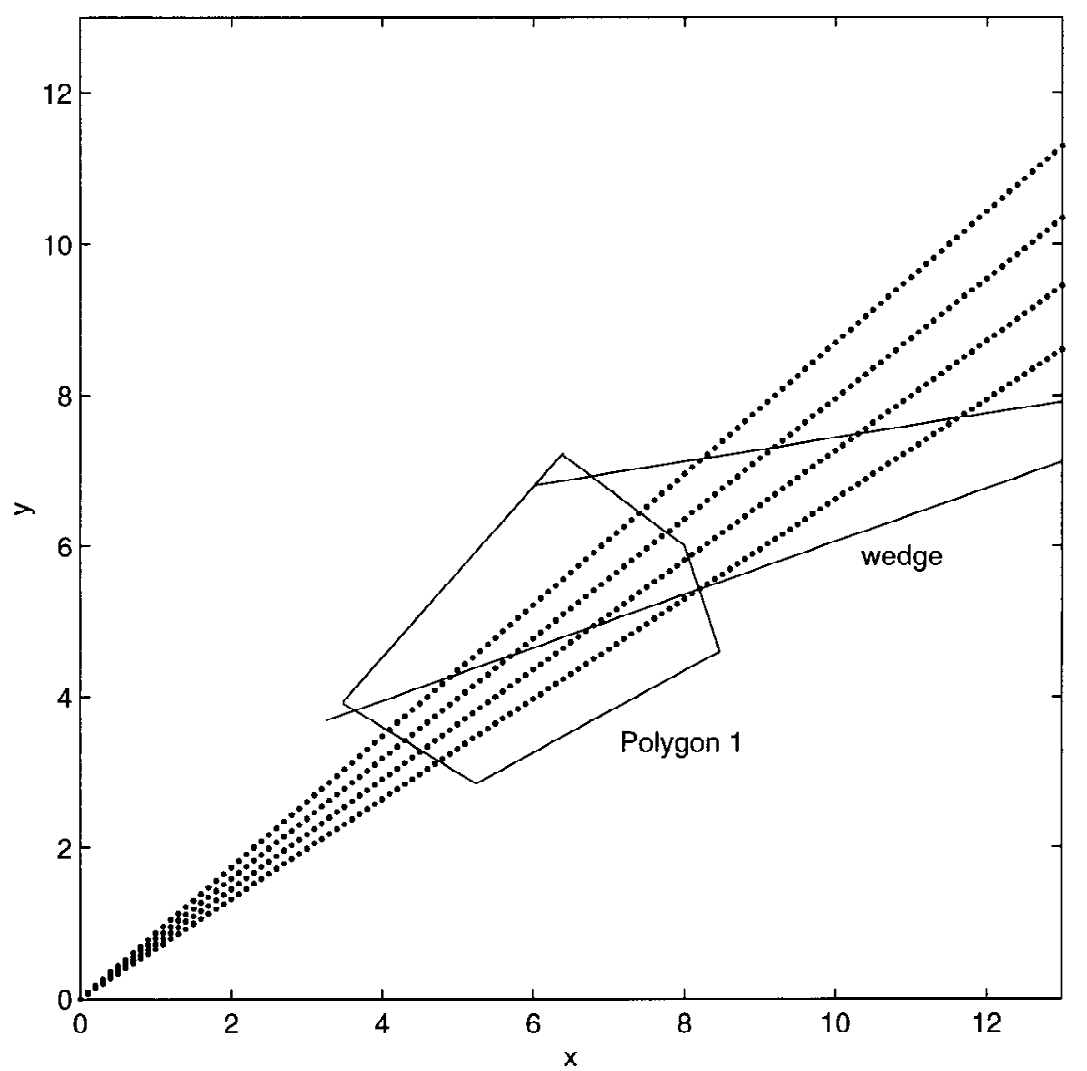

Fig. 4. Polygon 1 and a wedge with equal directed $X$-ray data along four rays. The four rays appear as dotted lines emanating from the origin.

$\sim 1.1459^{\circ}$. The first ray meeting the polygon was $\varphi=0.80$ radians and the last ray was $\varphi=2.42$ radians. Figure 6(a) shows a graph of the directed X-ray data of Polygon 2. The divided difference approximation to the difference of the left and right derivatives appears on the right. The jumps in the data in Fig. 6(b) locate rays between which vertices should lie. This divides the data set into four distinct pieces and Theorem 6.6 confirms that each piece comes from a nonparallel wedge. Polygon 2 and the reconstructed points $(+)$ are given in Fig. 7.

Example 7.3 Polygon 3 is more complicated. Figure 8(a) shows a graph of the directed $\mathrm{X}$-ray data. The data near the origin indicate that we are probably in the generic case where both supporting rays from the source meet the convex body in a single point. Here $\Delta=0.005$ radians $\sim 0.2865^{\circ}$. Computation of $Q\left(X_{j}, X_{j+1}, X_{j+2}\right)$ reveals that the polygon contains a parallel wedge as this value is on the order of $(10)^{-15}$ for the 13 rays in the cone $C(1.43,1.48)$. Figure 8 (b) shows a graph of the divided difference approximation to $X_{-}^{\prime}\left(\varphi_{0}\right)-X_{+}^{\prime}\left(\varphi_{0}\right)$. (Five points in from the far left but not visible 


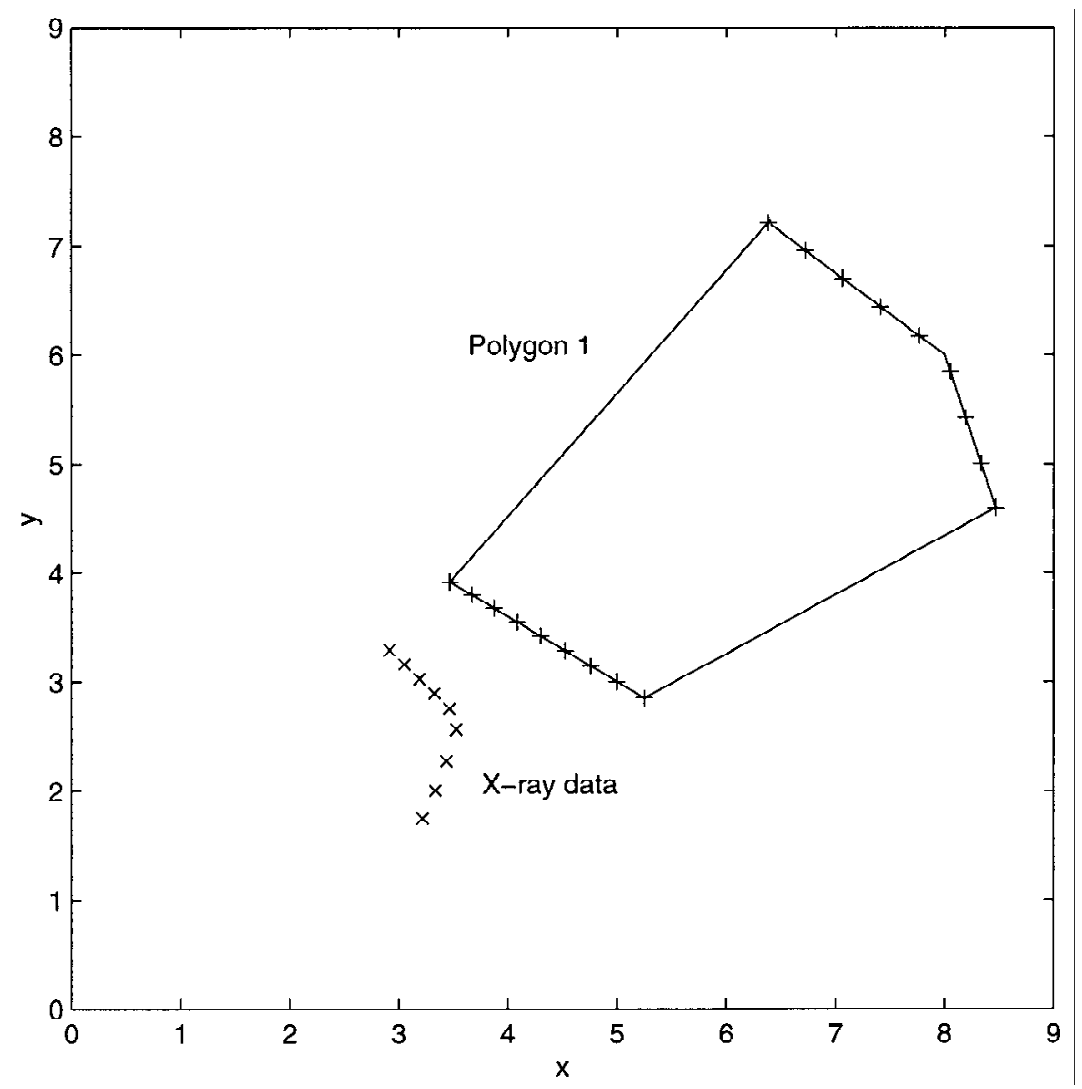

Fig. 5. Polygon 1 and overlaid reconstruction points $(+) . \times$, Directed $X$-ray data.

there is a point with value about 70 . The range chosen for the graph excluded this point. Including it would have masked smaller jumps.) From Fig. 8(b) we see that (excluding supporting rays) the polygon has nine or ten rays along which nonsmooth points lie. The cluster of four jumps near 1.2 radians indicates that there may be a feature in the polygon that is too fine to reconstruct exactly from our data. That is, there may be a small wedge which meets fewer than four rays in the data set. After approximately locating rays on which vertices occur, we proceed as in the previous examples and reconstruct nonparallel wedges where we have four or more data points. (When it is confirmed that a large set of rays are consistent with being from a single wedge, the reconstruction is done by selecting four rays with the angle between consecutive rays in the set as large as possible. This reduces possible loss of significance errors in the computations.) Polygon 3 and overlaid reconstructed points $(+)$ are shown in Fig. 9. The small parallel wedge is visible between rays making angles between 1.43 and 1.48 radians $\left(\sim 83^{\circ}\right)$. Polygon 3 has twelve sides and vertices at $(1,11),(5,12),(10,14),(11,18),(12,16),(8,20),(5,21)$, $(3,21),(-1,20),(-3,17),(-4,15)$, and $(-4,13)$. These vertices divide the polygon into ten wedges. One wedge is a parallel wedge lying between $\arctan (7) \sim 1.4289$ 


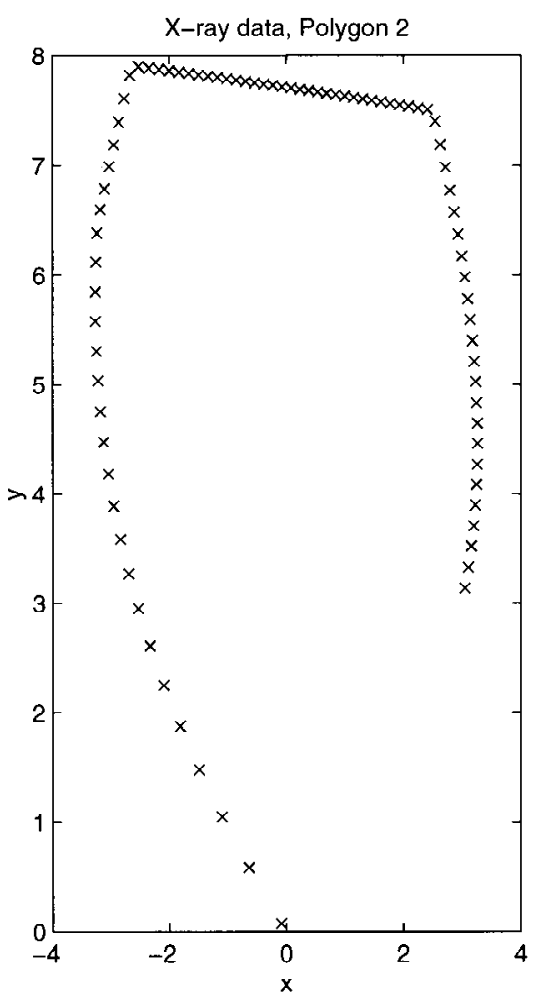

(a)

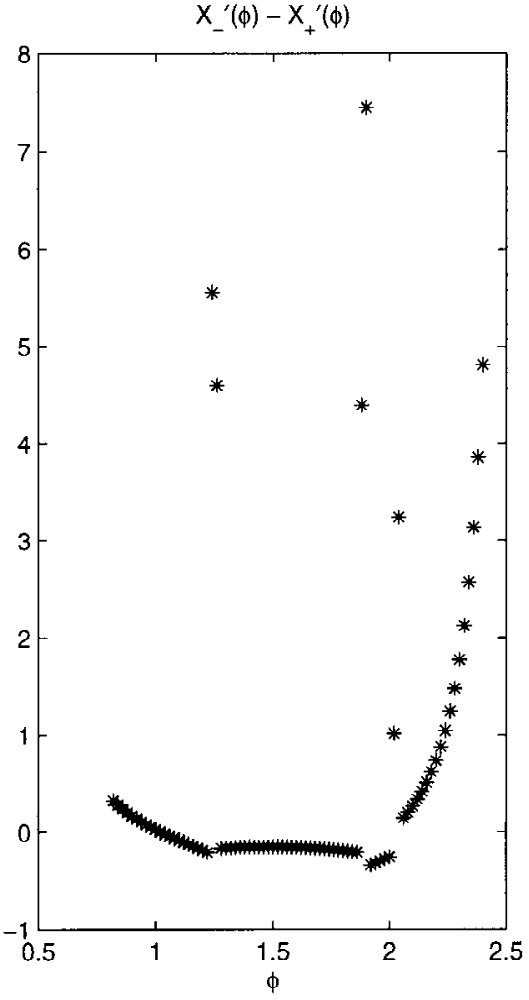

(b)

Fig. 6. (a) Directed X-ray data of Polygon 2. (b) Divided difference approximation to difference in left and right derivatives of directed X-ray for Polygon 2. Jumps indicate approximate location of vertices.

radians $\sim 81.87^{\circ}$ and $\arctan (11) \sim 1.4801$ radians $\sim 84.81^{\circ}$. Also, the vertices at $(8,20)$ and $(12,5)$ lie along rays making angles $\arctan \left(\frac{5}{2}\right) \sim 1.19028 \sim 69.1986^{\circ}$ and $\arctan \left(\frac{12}{5}\right) \sim 1.17601$ radians $\sim 67.3801^{\circ}$. With a ray sampling of 0.005 radians there are only three data rays in this wedge. A small gap appears in the reconstructed points corresponding to these three rays. Another feature of Polygon 3 is that its boundary contains a vertical line. It is surprising that this was reconstructed accurately without rotating the data. In this reconstruction Matlab computed a slope on the order of $10^{24}$. In an earlier reconstruction of the same polygon [13], the vertical feature was not reconstructed accurately. We do not know what accounts for the difference, but feel it is advisable to rotate the data before finding the point $r_{1}$ when $k_{1} k_{5}-k_{2} k_{4} \sim 0$. This avoids dividing by numbers that are almost zero.

Example 7.4. Figure 10(a) shows the directed X-ray data of Polygon 4. This time $\Delta=0.01$ radians $\sim 0.5730^{\circ}$ and the nonzero values lie in the cone $C(1.35,1.79)$. The data suggest that the supporting rays meet the polygon in a single point. The divided difference approximations to $X_{-}^{\prime}\left(\varphi_{0}\right)-X_{+}^{\prime}\left(\varphi_{0}\right)$ appear in Fig. 10(b). These indicate that 


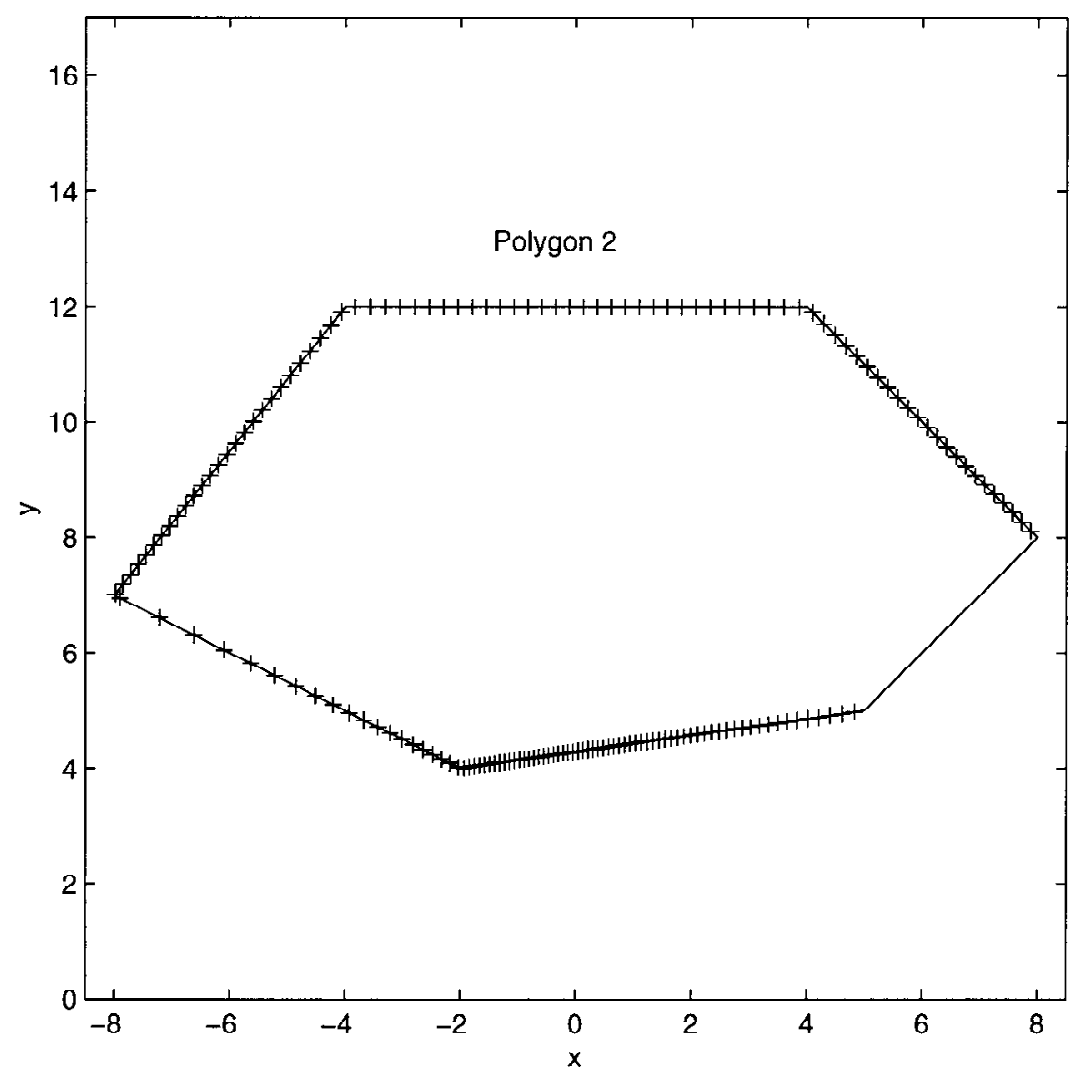

Fig. 7. Polygon 2 and overlaid reconstructed points (+).

there are two rays along which vertices lie, excluding the supporting rays, and that the polygon can be divided into three wedges. Theorem 6.6 shows that the first six data points come from a common wedge lying in the cone $C(1.35,1.40)$. Similarly, the last six come from a wedge in the cone $C(1.74,1.79)$. However, $(6.2)$ is not satisfied by the data points corresponding to rays near $\varphi=\pi / 2$. So the smooth middle section of data in Fig. 10 does not come from a single wedge. Using (6.8) to check for finite data sets that lie on a wedge we find that the middle section of data is consistent with coming from two nonparallel wedges, one located in the cone $C(1.41,1.57)$ and the other in the cone $C(1.59,1.73)$. Typically (6.8) gave values of around $10^{-17}$ for five consecutive data points from these intervals.

Polygon 4, which is not convex, and the reconstructed points are shown in Fig. 11. The polygon is bounded above by lines $3 y-4 x=18,3 y+4 x=18$, and below by lines $3 y-8 x=10, y-2 x=4, y+2 x=4$, and $3 y+8 x=10$. The vertices are $(0,4),(0,6),\left(2, \frac{26}{3}\right),\left(-2, \frac{26}{3}\right),(1,6)$, and $(-1,6)$. These lie along rays corresponding to angles $\arctan \left(\frac{13}{3}\right) \sim 1.34$ radians $\sim 77.501^{\circ}, \arctan (6) \sim 1.406$ radians $\sim 80.54^{\circ}, \pi-$ $\arctan (6) \sim 1.7359$ radians $\sim 99.46^{\circ}$, and $\pi-\arctan \left(\frac{13}{3}\right) \sim 1.798$ radians $\sim 102.99^{\circ}$. 


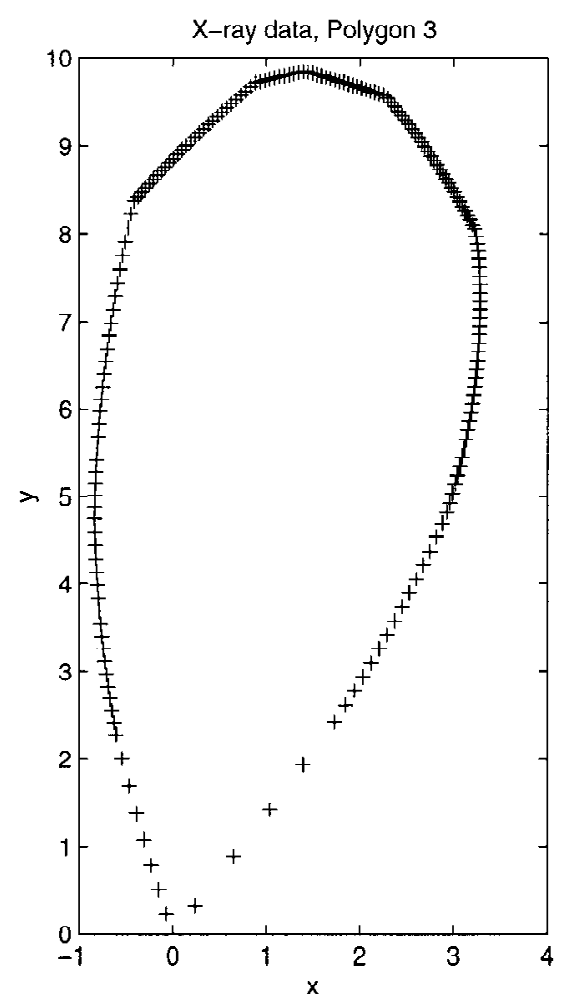

(a)

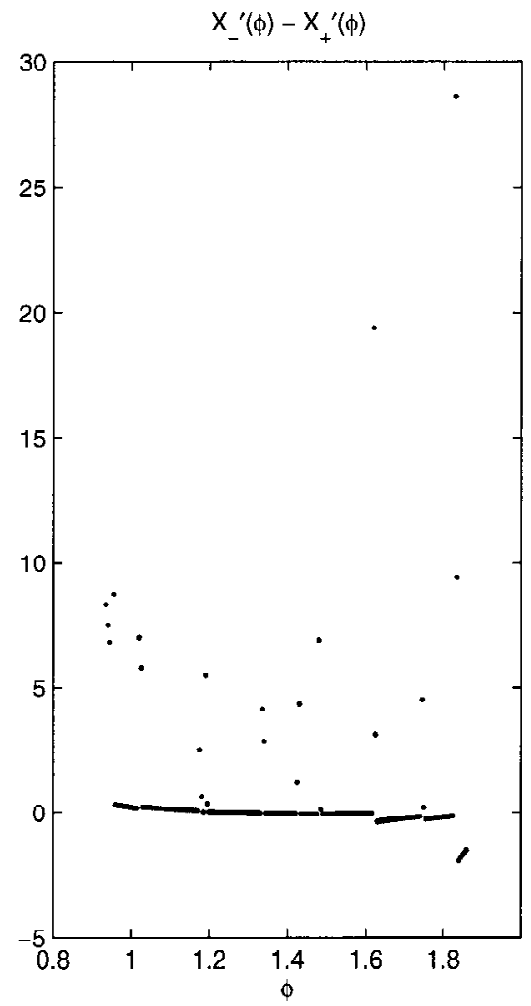

(b)

Fig. 8. (a) Directed X-ray data of Polygon 3. (b) Divided difference approximation to difference in left and right derivatives of directed X-ray for Polygon 3. Jumps indicate approximate location of vertices.

The vertices at $(0,4)$ and $(0,6)$ lie along the ray $\varphi=\pi / 2$. The slopes on the left and right at $(0,4)$ are -2 and 2 , respectively, while at $(0,6)$ the slopes are $-\frac{4}{3}$ and $\frac{4}{3}$, respectively. Returning to (2.5) we see that

$$
\begin{aligned}
\left(\mathcal{D} \chi_{K}\right)_{-}^{\prime}(\pi / 2) & -\left(\mathcal{D} \chi_{K}\right)_{+}^{\prime}(\pi / 2) \\
= & R(\pi / 2)\left[F_{-}^{\prime}(0)-F_{+}^{\prime}(0)\right]+r(\pi / 2)\left[f_{+}^{\prime}(0)-f_{-}^{\prime}(0)\right] \\
= & 6\left[-\frac{4}{3}-\frac{4}{3}\right]+4[2-(-2)]=-16+16=0 .
\end{aligned}
$$

This explains why the directed X-ray data and the divided differences in Fig. 10 are smooth in a neighborhood of $\varphi=\pi / 2$ even though there were vertices along that ray.

The discrete directed X-ray data did not contain the ray $\varphi=\pi / 2$. This can be seen in Fig. 10(a) as the X-ray data are not symmetric with respect to the vertical axis. However, $\pi / 2 \sim 1.5708$. With discrete data in steps of 0.01 radians, data were taken along the rays $\varphi=1.57$ and $\varphi=1.58$.

Remark 7.5. One can show that the directed X-ray of the polygon in Example 7.4 is $C^{2}$ but not $C^{3}$ in an open interval containing $\varphi=\pi / 2$. Lauzon and Schmidt [15] have 


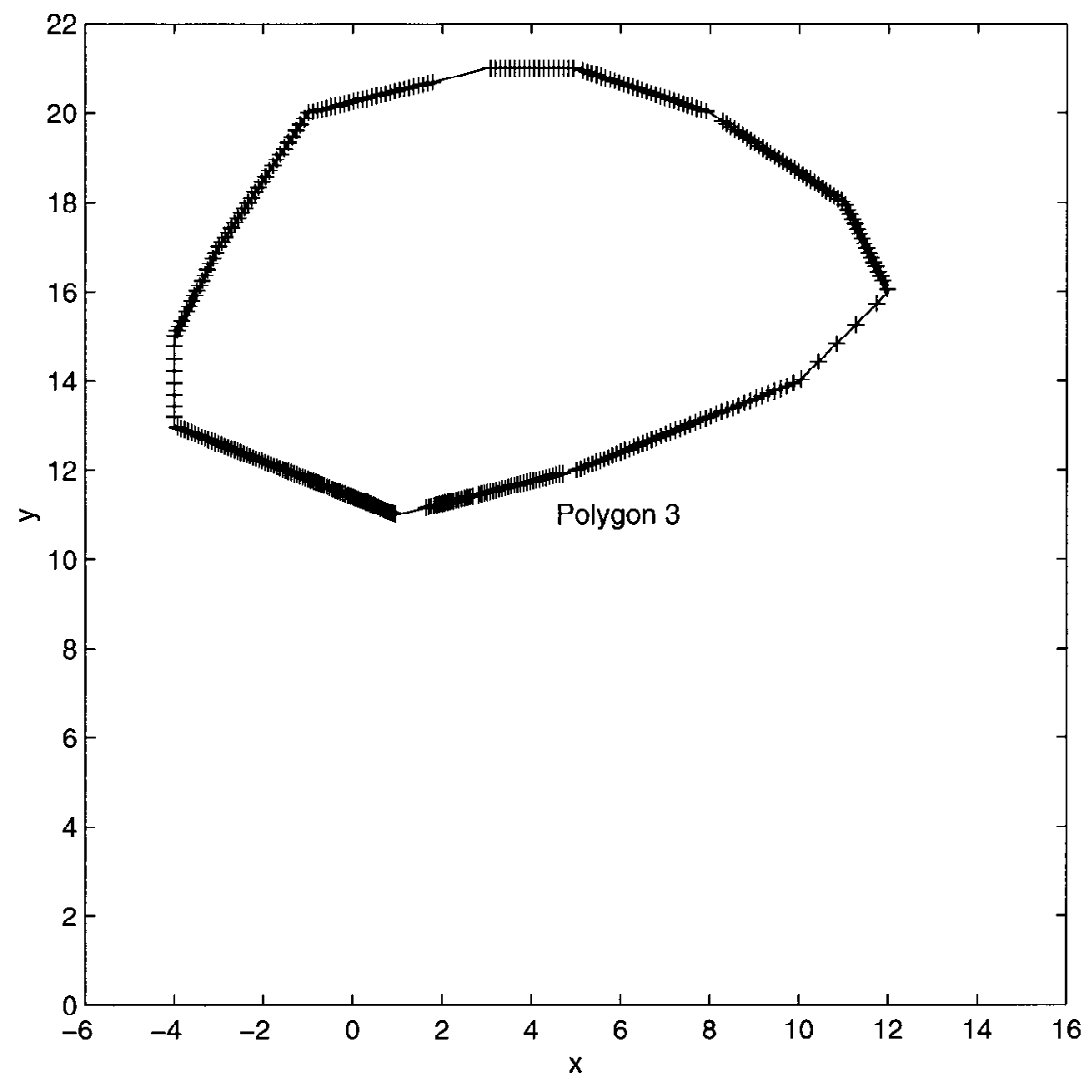

Fig. 9. Polygon 3 and overlaid reconstructed points (+).

shown that a nonparallel wedge is uniquely determined by its directed X-ray at a point $\varphi_{0}$ together with the first three derivatives of the directed X-ray at $\varphi_{0}$. Hence, vertices of polygons that are star-shaped with respect to the source may be located by finding discontinuities in one of the first three derivatives of the directed X-ray (or as was done in Example 7.4). The results of Section 4 or 5 can then be used to reconstruct nonparallel wedges determined by the vertices. Polygons which are star-shaped with respect to the source may contain more than one parallel wedge. Theorem 3.1 can be used to determine whether such polygons contain parallel wedges, but the position of any parallel wedge cannot be located exactly.

\section{Acknowledgments}

This work began during the NSF sponsored Research Experience for Undergraduates (REU) program during summer 1998 at Oregon State University. Preliminary results appeared in [14]. We thank Andrea Perrine who participated in the REU program and 


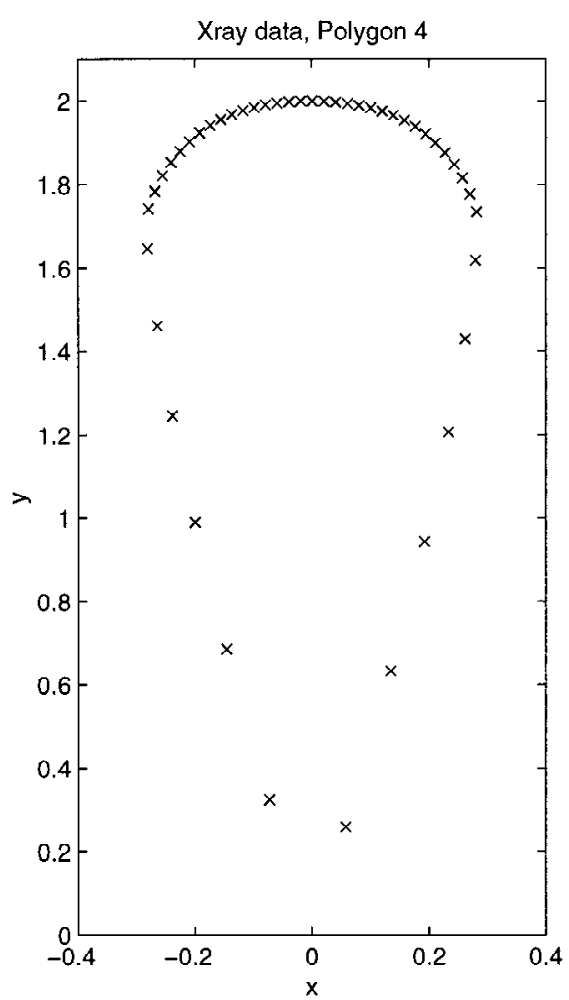

(a)

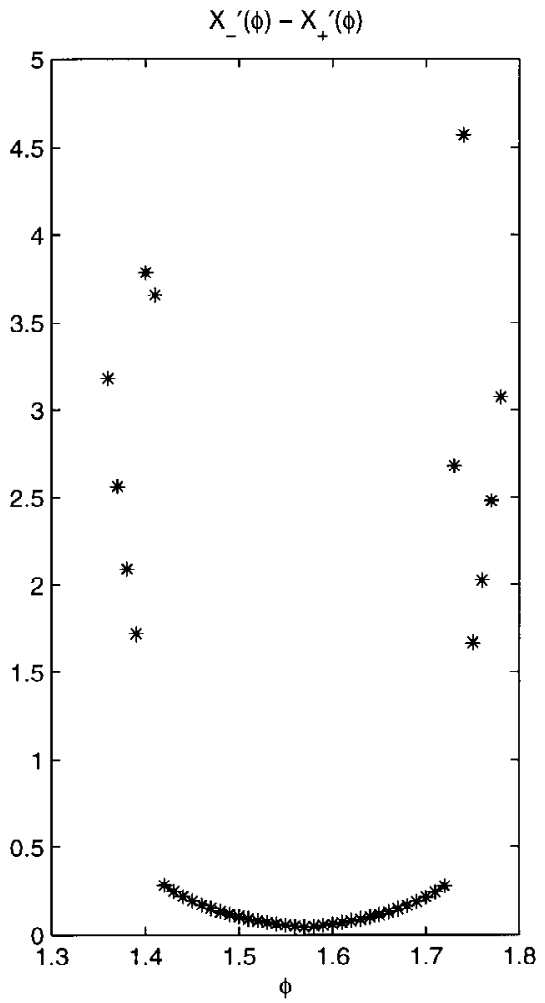

(b)

Fig. 10. (a) Directed X-ray data of Polygon 4. (b) Divided difference approximation to difference in left and right derivatives of directed X-ray for Polygon 4. Jumps indicate approximate location of vertices.

contributed to Sections 2 and 3. Related results and some of the results here appeared in the first author's Honor's Thesis at Wellesley College [13]. The first author thanks Professor Frederic Shultz of the Wellesley College Mathematics Department for his continuous support and encouragement with this project. His countless hours of teaching and advising are greatly appreciated.

The authors also thank the referee who provided a thorough critique of the paper and many helpful suggestions.

\section{Appendix. A Nonlinear Least Squares Algorithm}

Below is a Matlab program, LSQ.m, for the least squares algorithm outlined in Section 4. Comments inserted in the program are marked $\%$.

function $[r a, r b, R a, R b]=\operatorname{LSQ}(A, B, r a, r b, R a, R b)$

$\% A=$ initial angle, $B=$ terminal angle (in radians). 


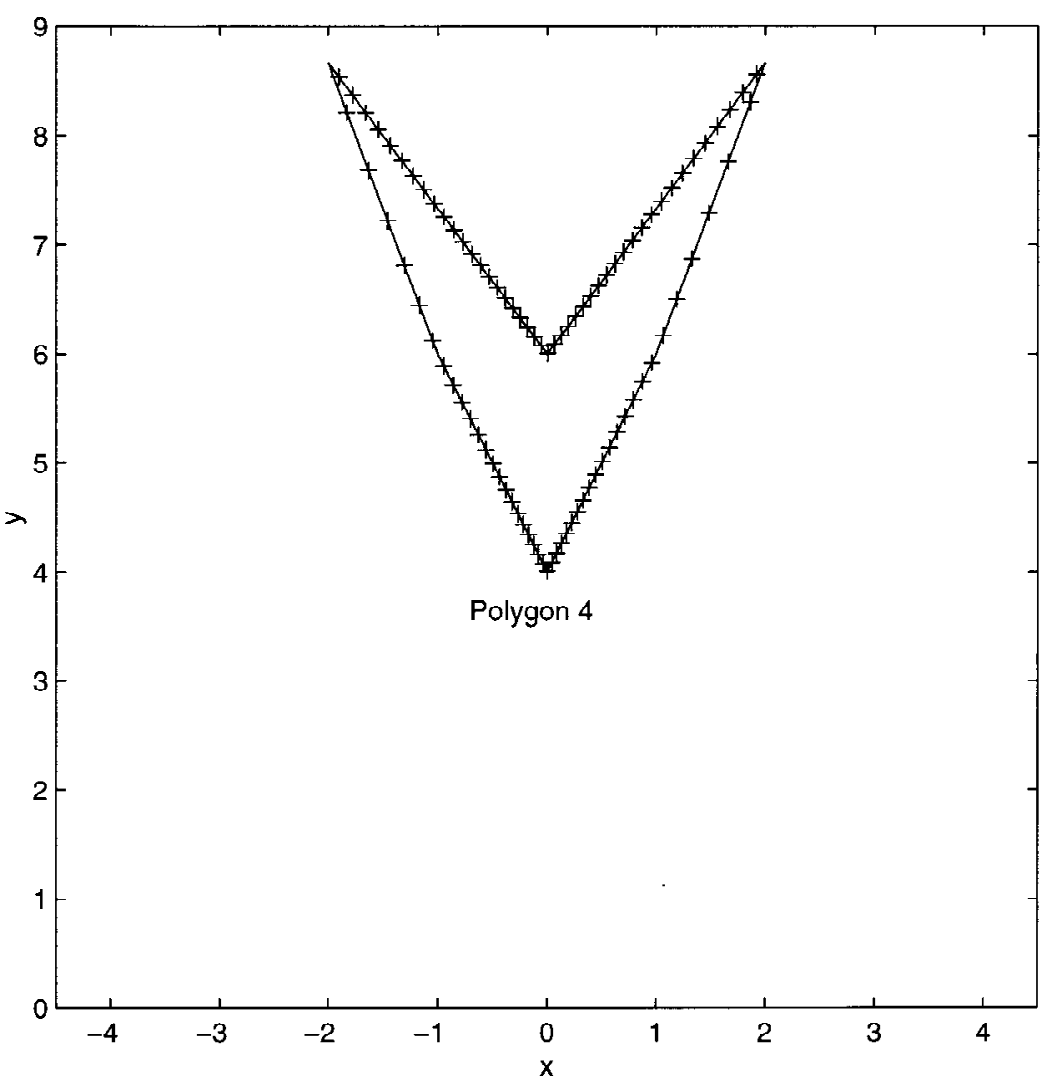

Fig. 11. Polygon 4 and overlaid reconstructed points $(+)$. Note that Polygon 4 has vertices where the data in Fig. 10 is smooth.

$\% r a, r b, R a, R b$ are the initial estimates for $r_{\alpha}, r_{\beta}, R_{\alpha}, R_{\beta}$.

$$
\begin{aligned}
p 1 & =\sin (B) / r a-\sin (A) / r b ; & q 1 & =\cos (A) / r b-\cos (B) / r a ; \\
r 1 & =\sin (B) / R a-\sin (A) / R b ; & s 1 & =\cos (A) / R b-\cos (B) / R a
\end{aligned}
$$

$[P]=$ leastsq(“vertices", $[p 1, q 1, r 1, s 1])$;

$\%$ The above statement calls the Matlab nonlinear least squares subroutine which min$\%$ imizes the function $E$ in the program "vertices" over the variables $p 1, q 1, r 1, s 1$.

$\%$ The minimizing values are returned as the vector $P$. A copy of "vertices" appears $\%$ below.

$$
\begin{gathered}
p f=P(1) ; \quad q f=P(2) ; \quad r f=P(3) ; \quad s f=P(4) ; \\
r a=\sin (B-A) /(p f * \cos (A)+q f * \sin (A)) \\
r b=\sin (B-A) /(p f * \cos (B)+q f * \sin (B))
\end{gathered}
$$




$$
\begin{aligned}
& R a=\sin (B-A) /(r f * \cos (A)+s f * \sin (A)) \\
& R b=\sin (B-A) /(r f * \cos (B)+s f * \sin (B))
\end{aligned}
$$

$\%$ The least square solutions are returned as $r a, r b, R a, R b$.

function $E=$ vertices (data)

$\%$ The data input to vertices are the values of $p 1, q 1, r 1, s 1$ computed from LSQ.m.

$$
p 1=\operatorname{data}(1), \quad p 2=\operatorname{data}(2), \quad p 3=\operatorname{data}(3), \quad p 4=\operatorname{data}(4)
$$

$\%$ The next two lines compute the left-hand side of (4.16), the modified X-ray data. The

$\%$ data here is from the first wedge of Polygon 1. See Example 7.1.

$$
\begin{gathered}
t=0.49: 0.01: 0.65 ; \quad L=\operatorname{ones}(1, \text { length }(t)) \\
R=30 * . /(\sin (t)+3 * \cos (t)) ; \quad r=30 * L . /(5 * \sin (t)+3 * \cos (t)) \\
M X R=(R-r) / \sin (0.16) ; \\
E=M X R-L . / r 1 * \cos (t)+s 1 * \sin (t))+L . /(p 1 * \cos (t)+q 1 * \sin (t))
\end{gathered}
$$

$\% E$ is the function minimized by the Matlab least square subroutine. See (4.16).

\section{References}

1. S.-K. Chang and C. K. Chow, The reconstruction of three-dimensional objects from two orthogonal projections and its application to cardiac cineangiography. IEEE Trans. Comput. 22 (1973), 18-28.

2. S.-K. Chang and Y. R. Wang, Three-dimensional object reconstruction from orthogonal projections. In Pattern Recognition. Pergamon Press, Oxford, 1975, pp. 167-176.

3. M. Dartmann, Rekonstructionen konvexer, homogener Gebeite aus wenigen Punktquellen. Diplomarbeit, University of Münster, Münster, 1991.

4. H. Edelsbrunner and S. S. Skiena, Probing convex polygons with x-rays. SIAM J. Comput 17 (1988), 870-882.

5. K. J. Falconer, X-ray problems for point sources. Proc. London Math. Soc. (3) 46 (1983), 241-262.

6. K. J. Falconer, Hammer's X-ray problem and the stable manifold theorem. J. London Math. Soc. (2) 28 (1983), 149-160.

7. R. J. Gardner, Symmetrals and X-rays of planar convex bodies. Arch. Math. 41 (1983), 183-189.

8. R. J. Gardner, X-rays of polygons. Discrete Comput. Geom. 7 (1992), 281-293.

9. R. J. Gardner, Geometric Tomography. Encyclopedia of Mathematics and its Applications, vol. 58. Cambridge University Press, Cambridge, 1995.

10. R. J. Gardner and P. McMullen, On Hammer's X-ray problem. J. London Math. Soc. (2) 21 (1980), $171-125$.

11. P. C. Hammer, Problem 2. In Convexity. Proceedings of Symposia in Pure Mathematics, vol. VII. American Mathematical Society, Providence, RI, 1963, pp. 498-499.

12. D. Kölzow, A. Kuba, and A. Volčič, An algorithm for reconstructing convex bodies from their projections. Discrete. Comput. Geom. 4 (1989), 205-237.

13. D. Lam, Point source geometric tomography. Honors Thesis, Wellesley College.

14. D. Lam and A. Perrine, Point source geometric tomography. Proceedings of the REU Program in Mathematics, Oregon State University, Summer 1998, pp. 101-121. 
15. M. Lauzon and B. Schmidt, Tomography of non-convex polygons from a single point source. Proceedings of the REU Program in Mathematics, Oregon State University, Summer 1999, pp. 77-99.

16. M. Longinetti, Some questions of stability in the reconstruction of plane convex bodies from projections. Inverse Problems 1 (1985), 87-97.

17. H. L. Royden, Real Analysis, third edition. Macmillan, New York, 1988.

18. A. Volčič, A three-point solution to Hammer's X-ray problem. J. London Math. Soc. (2) 40 (1989), $171-178$.

Received August 16, 1999, and in revised form September 13, 2000. Online publication May 4, 2001. 